\title{
From molecular chemistry to supramolecular chemistry to superdupermolecular chemistry. Controlling covalent bond formation through non-covalent and magnetic interactions
}

\author{
Nicholas J. Turro \\ Chemistry Department, Columbia University, New York, NY 10027, USA. E-mail: turro@chem.columbia.edu
}

Received (in Cambridge, UK) 7th June 2002, Accepted 23rd July 2002

First published as an Advance Article on the web 6th September 2002

\begin{abstract}
The reactions of carbon centered radical pairs often involve diffusion controlled combination and/or disproportionation reactions which are non-selective. A triplet geminate pair of radicals is produced by the photolysis of suitable ketones. The reactions of such geminate pairs can be controlled though the application of supramolecular concepts which emphasize noncovalent interaction to "steer" the geminate pair toward a selected pathway. In addition, "superdupermolecular" concepts, which emphasize the control of radical pair reactions through the orientation of electron spins, can be employed to further control the course of geminate pair reactions. Examples of control of a range of the selectivity of geminate radical combinations, which form strong covalent bonds, through supramolecular and superdupermolecular effects will be presented for the photolysis of ketones adsorbed in the supercages of zeolites.
\end{abstract}

\section{Introduction}

Carbon centered radicals are among the most reactive species encountered in organic chemistry. The molecular chemistry of radical pairs is often dominated by diffusion controlled, random and non-selective radical-radical reactions (combinations and disproportionations). This report describes the application of the concepts of supramolecular chemistry (chemistry that is dominated by forces resulting from intermolecular non-bonded, non-covalent electrostatic forces (due to static and oscillating

Nicholas J. Turro is the William P. Schweitzer Professor of Chemistry at Columbia University in New York City. He received a $B A$ from Wesleyan University, a PhD from Caltech with George Hammond and spent a postdoctoral year at Harvard with Paul Bartlett before taking a position at Columbia University in 1964. His research interests have involve the use of photochemistry and spectroscopy to investigate the structure and dynamics of molecular and supramolecular systems. He has always been fascinated by the synthesis of organic reactive intermediates and their involvement in reaction mechanisms. Most recently his research interests have included on the mechanism of free radical polymerization, the mechanisms of spin effects on radical pair reactivity, the characterization of dendrimers, the use of time resolved EPR to investigate radicals produced in molecular and supramolecular systems and the photochemistry of organic molecules adsorbed on zeolites. He is a member of the National Academy of the USA and was awarded the Colloids and Surface Chemistry Award by the American Chemical Society, the Gibbs Medal of the Chicago Section of the American Chemical Society. Most recently he received a National Science Foundation Director's Distinguished Teaching Scholar Award. fixed charge interactions) and dispersion forces (due to induced transient charge interactions) and of superdupermolecular chemistry (chemistry that is dominated by forces due to magnetic interactions resulting from electron and nuclear spins and applied magnetic fields) to control the selectivity of reactions of geminate radical pairs whose molecular chemistry involves random radical-radical reactions.

Examples will be presented from the author's laboratory to demonstrate how the stereoselectivity, regioselectivity, chemioselectivity, geminatoselectivity and magnetoselectivity of geminate radical pair chemistry can all be controlled by creation of a geminate radical pair in a zeolite supercage through photochemical excitation of a ketone@supercage complex and by manipulation of supramolecular and superdupermolecular structural features that are at the disposal of the experimentalist.

It is important to calibrate for the reader the magnitude 'strength' or 'weakness' of the supramolecular electrostatic interactions and the superdupermolecular magnetic interactions, and to compare them to the more familiar strength of covalent bonds, which range from $c a$. 30-100 kcal mole ${ }^{-1}$. Supramolecular bonds are generally much weaker. A typical range for supramolecular intermolecular bonds is $c a .5 \mathrm{kcal}$ mole $^{-1}$ for an individual hydrogen bond to much less than $k T$ (ca. $1 \mathrm{kcal} \mathrm{mole}^{-1}$ ) for intermolecular bonds due to dispersion forces. The range of superdupermolecular magnetic 'bonds' or interactions is far smaller by comparison. Even for very strong magnetic fields ( $c a .100000$ gauss) the energy associated with the field acting on an electron spin is of the order of $1 \times 10^{-2}$ $\mathrm{kcal} \mathrm{mole}^{-1}$. The value for magnetic interactions of a field of 100000 gauss with a nuclear spin is only $c a .1 \times 10^{-5} \mathrm{kcal}$ mole ${ }^{-1}$ or less. In spite of the tiny value of these magnetic forces, we shall show that they can control the reactivity of radical pairs in a spectacular manner if the supramolecular conditions are correct!

\section{The origins of supramolecular chemistry. The 'cage effect' from the solvent cage to the supercage}

The starting point in the history of supramolecular effects on the chemistry of geminate radical pairs may be traced back to a publication $^{1}$ which coined the term 'cage effect' to explain observations comparing the photochemistry of diatomic molecules $\left(e . g ., \mathrm{I}_{2}\right)$ in the gas phase to their photochemistry in the liquid phase:

"Even though a molecule in solution may happen to dissociate after light absorption, and the radicals or atoms formed in this way separate with a certain amount of kinetic energy, this excess energy will be at once lost in collisions with the solvent. In addition to the 'normal' probability of recombination governed by the law of mass action, there will be 
an additional probability of primary recombination of two particles which have been parts of the same molecule before dissociation...The recombination effect will probably show a wavelength dependence, decreasing with the increasing energy of the absorbed quantum. A greater excess energy will permit the dissociation products to find their way through the surrounding 'walls' of the solvent and to put more molecular layers between them before coming to rest...Compared to the gas phase, the probability of a recombination of a reactive pair of fragments produced by photodissociation is lower in solution because of the action of the 'solvent cage' about the pair".

A surface energy description of the idea of the cage effect is shown in Fig. 1 (left). The brilliant insight and imagery of the importance of a radical pair in a 'solvent cage' may be considered as setting the stage for supramolecular chemistry, ${ }^{2}$ which is concerned with how non-covalent, intermolecular interactions can influence chemistry of 'bimolecular' systems. The solvent cage is a primitive but fundamental supramolecular 'host' that exerts an influence on the chemistry and reactivity of an incarcerated 'guest' molecule or pair of 'guest' molecules. ${ }^{3}$ This report will use the metaphor of guest@host chemistry to inspire the design systems for which the chemistry of a 'guest' radical pair is controlled by a 'supercage' which is an extension of the idea of the solvent cage. ${ }^{1}$

\section{Collisions in a solvent cage occur in sets. Fundamental characteristics of a supercage host: multiple collisions and multiple encounters of 'caged' guest molecules}

Two years after the concept of a solvent cage ${ }^{1}$ appeared, an experiment ${ }^{4}$ using a mechanical device representing collisions between molecules demonstrated that, when two molecules that are surrounded by a solvent cage collide, the collisions between a neighboring pair of molecules occur in sets (Fig. 2, left). The occurrence of collision sets in a dense medium such as a liquid is due to the fact that the colliding pair of particles is surrounded by a 'cage' of solvent molecules and can escape from this 'cage' only by a process of diffusion during which the pair of neighbors collide a number of times. Pairs of radicals formed by a photochemical (or thermal) dissociation of a molecule are, immediately after their formation, in the same position as two dissolved particles after a collision. This situation offers the possibility of an immediate recombination of the dissociation

\section{Solvent Cage}

Super Cage
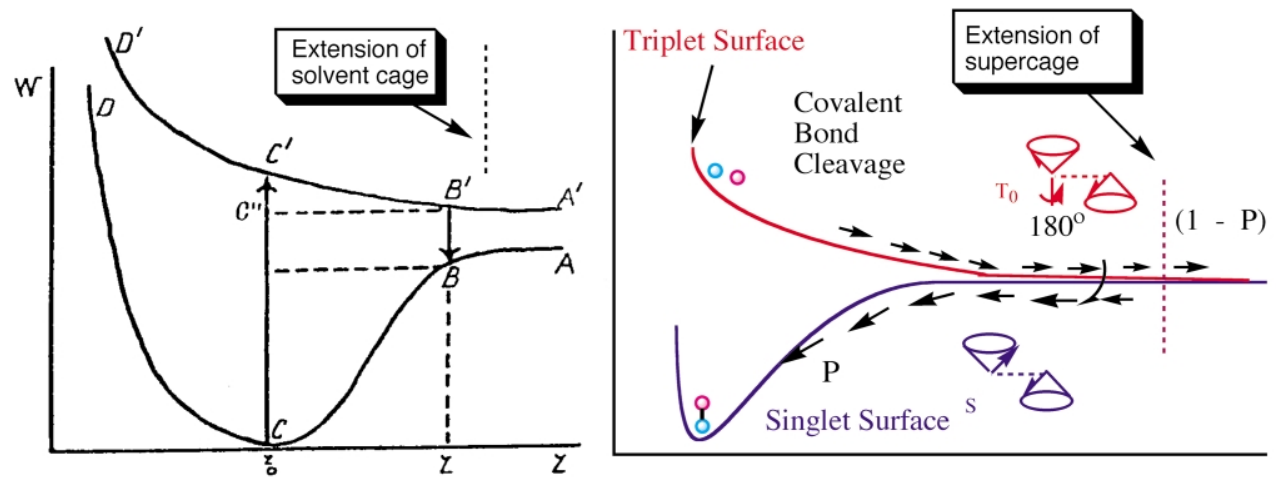

Fig. 1 The energy surface description proposed to understand the molecular cage effect (left). A modern description proposed to understand the molecular cage effect which includes electron spin. $P$ is the probability that a geminate pair will recombine and $(1-P)$ is the probability that some other irreversible reaction or process will occur.

Supramolecular Pin Ball Machine

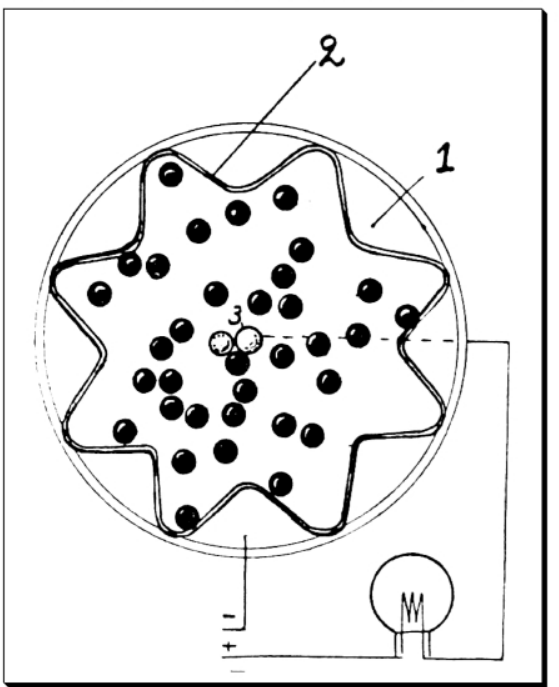

Superdupermolecular Spin Ball Machine

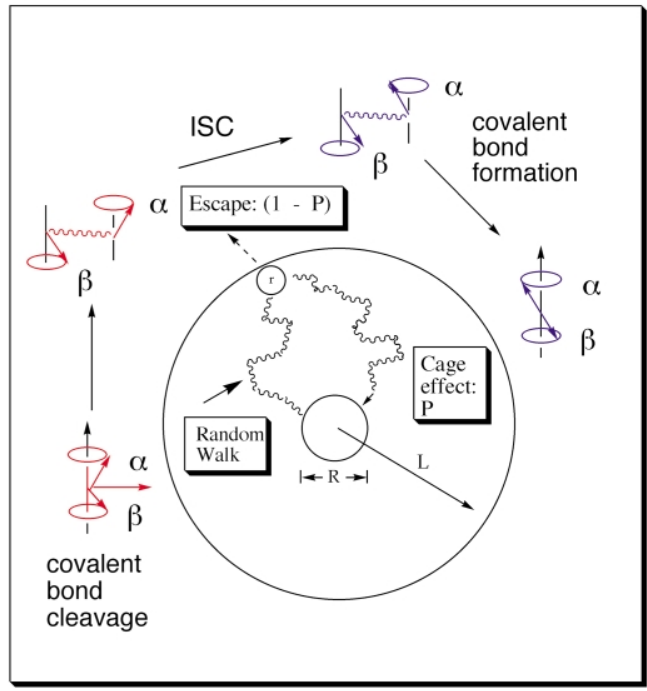

Fig. 2 The interplay of chemical dynamics, molecular dynamics and spin dynamics in a supercage. A supramolecular pinball machine (left) demonstrates how containment in a supramolecular cage leads to multiple collisions of neighboring particles in a 'supramolecular cage'. A superdupermolecular spin ball machine (right) demonstrates how containment and molecular dynamics in a supramolecular cage are coupled to electron spin and the critical intersystem crossing mechanism that converts an 'inert' triplet geminate radical pair into a 'reactive' singlet geminate radical pair. $P$ is the probability that a triplet geminate pair will recombine and $(1-P)$ is the probability that some other non-geminate, spin independent irreversible reaction or process will occur. 
products and is termed primary geminate recombination of the radical pair to distinguish it from 'random' recombination of radicals distributed at random throughout the whole body of the solution. The extent of geminate recombination is a measure of the 'cage effect' of the solvent walls to restrain diffusion of a pair of a 'geminate pair' of radicals that are 'born together' as the result of a photochemically induced dissociation. This mechanical device may be considered as a model of a supramolecular host or 'supercage' which contains a radical pair and which can control, through intermolecular interactions, the collisions and encounters of a radical pair.

\section{Reencounters of a geminate pair may occur even after a pair escapes from a solvent cage}

Even after the fragments of a geminate pair have separated by one or several solvent molecules, there may be a significant probability that the separated pair will reencounter one or more times. ${ }^{5}$ The reason for this is that two geminate fragments that have separated after an encounter remain so close that, in undergoing a random walk, there is a significant probability that they will make additional encounters with each other before they separate irreversibly and encounter radicals from other dissociations. The concepts of the cage effect ${ }^{1}$ (solvent walls as supramolecular host), the occurrence of collisions in sets (encounters), ${ }^{4}$ and the occurrence of encounters in sets (reencounters $)^{5}$ provide an exemplar for distinguishing a molecular cage from a supramolecular supercage: a supercage is defined as being capable of encouraging a much larger number of reencounters of a geminate pair than an ordinary molecular solvent cage.

\section{Geminate radical pairs, free radicals and random radical pairs}

Thermal or photochemical homolytic cleavage of a $\mathrm{C}-\mathrm{C}$ bond of a molecule in solution produces a pair of carbon centered radical fragments that are 'born together' (a 'geminate radical pair'). Geminate pairs typically undergo radical-radical combination (or disproportionation which we are ignoring in this account) reactions to form stable molecules or they undergo diffusional separation to form free radicals. When free radicals randomly encounter and become neighbors in a solvent cage, they are termed 'random radical pairs'. Random radical pairs may also undergo the same radical-radical combination reactions as geminate radical pairs. Thus, radical-radical combination reactions may be of two types: geminate and random. The term 'cage effect' or the probability, $P$, of geminate combination has been coined ${ }^{6}$ as a measure of the fraction of initial dissociation events that result in combination rather than free radical formation. We coin the term geminatoselectivity to describe the selectivity of an initial geminate pair for geminate combination relative to free radical formation.

In low viscosity solvents, ${ }^{7}$ the geminatoselectivity for the geminate radical-radical combination reactions resulting from the photolysis of ketones is typically close to $0 \%$, which will serve as a benchmark experimental signature for a molecular solvent cage. An experimental signature of a supramolecular cage (a supercage) will be the ability to enhance the magnitude of the geminatoselectivity of radical-radical reactions to values significantly above $0 \%$.

\section{Supramolecular chemistry. Guest @ host chemistry from the solvent cage to the supercage}

Supramolecular chemistry may be defined ${ }^{2}$ broadly as 'chemistry beyond the molecule'. ${ }^{2}$ Currently, the field appears to be mainly concerned with mastering the creation and manipulation of non-covalent bonding. Molecular structure and dynamics employ the key intellectual units ${ }^{8}$ of the valence of atoms and the covalent bond between atoms. We assume ${ }^{9}$ that supramolecular structure can be characterized at the static level by a composition (number and kinds of molecules), composition (connectivity relationships between molecules), configuration (local spatial relationships of molecules about one another) and conformation (global spatial relationships of the entire supramolecular assembly). Supramolecular dynamics involve both physical interconversions of conformational shapes and chemical reactions that convert one supramolecular structure into another. Thus, we take the point of view that the emerging science of supramolecular chemistry represents a natural extension of the science of molecular chemistry.

Guest@host supramolecular chemistry is of special interest to this report. Signature characteristics 2,3 of a 'classical' guest@host complex are: (1) the guest is a molecule whose size is small relative to the host, and (2) the host may be viewed as structurally providing a convex space that serves as a "container' for the guest and is accessible for binding the guest to a significant extent. A substrate@enzyme complex ${ }^{10}$ is an exemplarguest@host complex.

\section{The enzyme inspiration}

Natural enzymes catalyze the reactions of substrates with high selectivity at mild temperatures, but denature and undergo irreversible decomposition at elevated temperatures or under harsh acidic, basic, oxidative or reductive conditions. Thus, natural enzymes are not attractive for use in industrial processes that often require high temperature and hostile reaction conditions. In addition, enzymes are relatively expensive reagents and, therefore, are not attractive candidates for use on a massively large scale in industry. In contrast, zeolites are robust, inexpensive, tolerant to strong acidity and highly oxidizing conditions and serve as exceptionally stable heterogeneous catalysts. On the downside, zeolites possess an inferior catalytic selectivity as compared to enzymes.

The active site of an enzyme is a protein mantle, which may be either flexible or rigid, depending on the requirements of the reaction at hand. In zeolite-based, enzyme-inspired mimics, the function of the size-shape selective organic protein 'supercage' is replaced by the supercages formed by a rigid inorganic framework that creates the porous internal surface of a zeolite.

\section{How do enzymes deal with highly reactive intermediates? Zeolites as models of reaction selectivity by negative catalysis}

It has been postulated that in special cases that are 'chemically difficult' or 'improbable', enzymes may generate stabilized radicals which become involved in a catalytic pathway. ${ }^{11}$ Reaction selectivity in these cases may be achieved by a socalled 'negative catalysis' in which a 'hot' intermediate, such as a radical, is prevented from undergoing characteristic reactions that occur rapidly in solution, thereby prolonging the lifetime of the intermediate. With the 'default' solution reactions such as radical-radical combinations inhibited, the radical intermediate then has an opportunity to undergo reactions which are unusual when compared to those typically observed in solution. The term 'negative catalysis' is employed to mean that reaction selectivity is achieved by inhibiting undesired reactions rather than by accelerating target reactions.

The mechanisms of the catalytic action of the reaction of guest@zeolite complexes have a number of analogies to the mechanisms of the catalytic reaction of guest@enzyme complexes. ${ }^{12}$ For example, the guest is first adsorbed on the external 
surface of a zeolite particle and is sieved through diffusional processes into the internal surface to the active site. The zeolite framework provides a rigid host structure for binding of the guest. Because of the finite surface area, the initial binding sites on the external surface are limited in number and are well defined in terms of size/shape characteristics of binding a guest and of determining the diffusion of a guest from an initial binding site on the external surface to the internal surface. From the standpoint of basic scientific research, it is interesting to seek similarities of enzymes and zeolites and to seek ways to combine attractive features of the robust, chemically inert zeolite framework to mimic the ability of enzymes to control the selectivity of chemical reactions of guests adsorbed at active sites. In the enzyme the active site is created by the chemically active, protein environment, whereas for a zeolite the active site is created by the chemically inert, rigid aluminosilicate framework. The size/shape characteristics of the guest determine the diffusional properties of the guest as it moves towards the active site.

Thermal reactions catalyzed by zeolites are typically initiated as the result of adsorption of the guest to an active site of the host which possesses a strongly acidic functionality that causes certain critical bonds of the guest to be weakened and critical reactive intermediates to be formed. ${ }^{13}$ In this report we wish to avoid totally thermal initiation of the bond breaking process and we seek to operate with a guest in a chemically inert zeolite supercage. The formation of critical, reactive intermediates is achieved by the absorption of light by the adsorbed guest molecule, which undergoes a primary photochemical reaction to form a geminate radical pair. As a result, the 'active site' for formation of the reactive intermediate is determined by the initial supramolecular structure of the guest@ zeolite supramolecular complex. Let us now see how we can produce geminate radical pairs in the supercages of zeolites to form ketone@ zeolite complexes and then see how we can control the chemistry of the geminate pair through rational and systematic variations of supramolecular structure and dynamics.

\section{Exemplars for radical pair reactions of dibenzyl ketones}

Schemes 1 and 2 are specific exemplars to track the plausible fates of the radical pairs generated by photolysis of two guest geminate radical pair precursors: dibenzyl ketone (DBK) ${ }^{14}$ and 2,4-diphenylpentanone-2 (DPP), ${ }^{15}$ respectively. For each ketone, absorption of a photon causes $\alpha$-cleavage to produce a triplet primary geminate radical pair ${ }^{3} \mathrm{RP}-1$. After intersystem crossing (ISC) to a singlet geminate radical pair, ${ }^{1} \mathrm{RP}-1$, one of several carbon-carbon bond forming combination reactions, can occur: (a) recombination to re-form the bond that was cleaved to regenerate a structure identical to that of the parent ketone; (b) combination through coupling to one of the ortho positions (followed by a 1,3-H shift) to produce a regioisomer of the starting ketone; (c) combination by coupling to the para position (followed by a 1,3-H shift) to produce a regioisomer of the starting ketone. These reactions compete with loss of $\mathrm{CO}$ (ca. $100 \mathrm{~ns}$ for the pair from DBK and $c a .20 \mathrm{~ns}$ for the pair from DPP) ${ }^{15}$ from the triplet primary pair to produce the secondary geminate pair RP-2, presumably in the triplet state. As in the case of the primary pair, several combination reactions are possible for RP-2, but experimentally, only the combinations to form diphenylethanes (DPEs) from DBK or diphenylbutanes (DPBs) from DPP are observed to be significant. ${ }^{14,15}$ There is also a competition between geminate combination of RP-2 and irreversible diffusional separation of the geminate pair to form free radicals, FR. The FR eventually combine to form DPEs. All of these processes are characterized by a set of probability values, $P$, which will be defined below.

\section{The molecular photochemistry of DBK and DPP}

In non-viscous solutions photolysis of DBK or of DPP has been shown ${ }^{14,16,17}$ to produce only free radicals which couple to form DPE and DPB, respectively. There are no significant geminate primary or secondary pair combinations, i.e., the cage effect or probability of geminate reactions is close to zero, exactly the signature of a very weak molecular solvent cage. Thus, the paths RP-1 $\rightarrow$ RP-2 $\rightarrow$ FR $\rightarrow$ FR Coupling Products in Schemes 1 and 2 are exemplars of the molecular photochemistry of DBK and DPP, respectively. The evidence for the free radical nature of the reactions includes the ability to completely scavenge radicals ${ }^{14}$ produced by photolysis and the formation of a $1: 2: 1$ mixture of coupling products $\left(\mathrm{d}_{0}, \mathrm{~d}_{5}\right.$ and $\mathrm{d}_{10}$ DPE and DPB) when the deuterated ketones asym-DBK- $\mathrm{d}_{5}$ or $a s y m-\mathrm{DPP}-\mathrm{d}_{6}$ are photolyzed in solvents such as acetonitrile and benzene. ${ }^{17}$

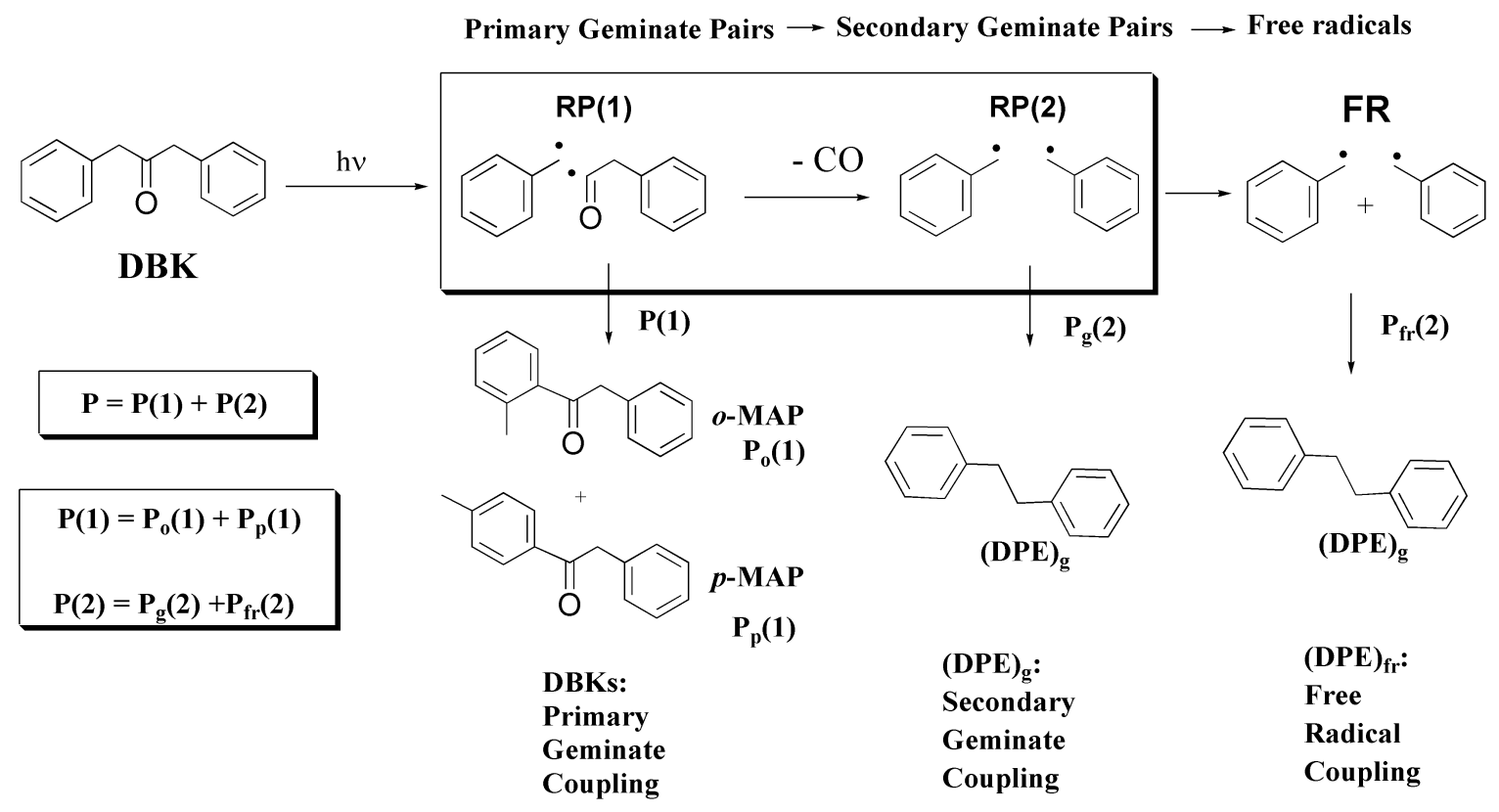

Scheme 1 Reaction mechanism and radical pair reaction pathways in the photochemistry of DBK. See text for discussion. 


\section{Primary Geminate Pairs $\rightarrow$ Secondary Geminate Pairs $\rightarrow$ Free radicals}

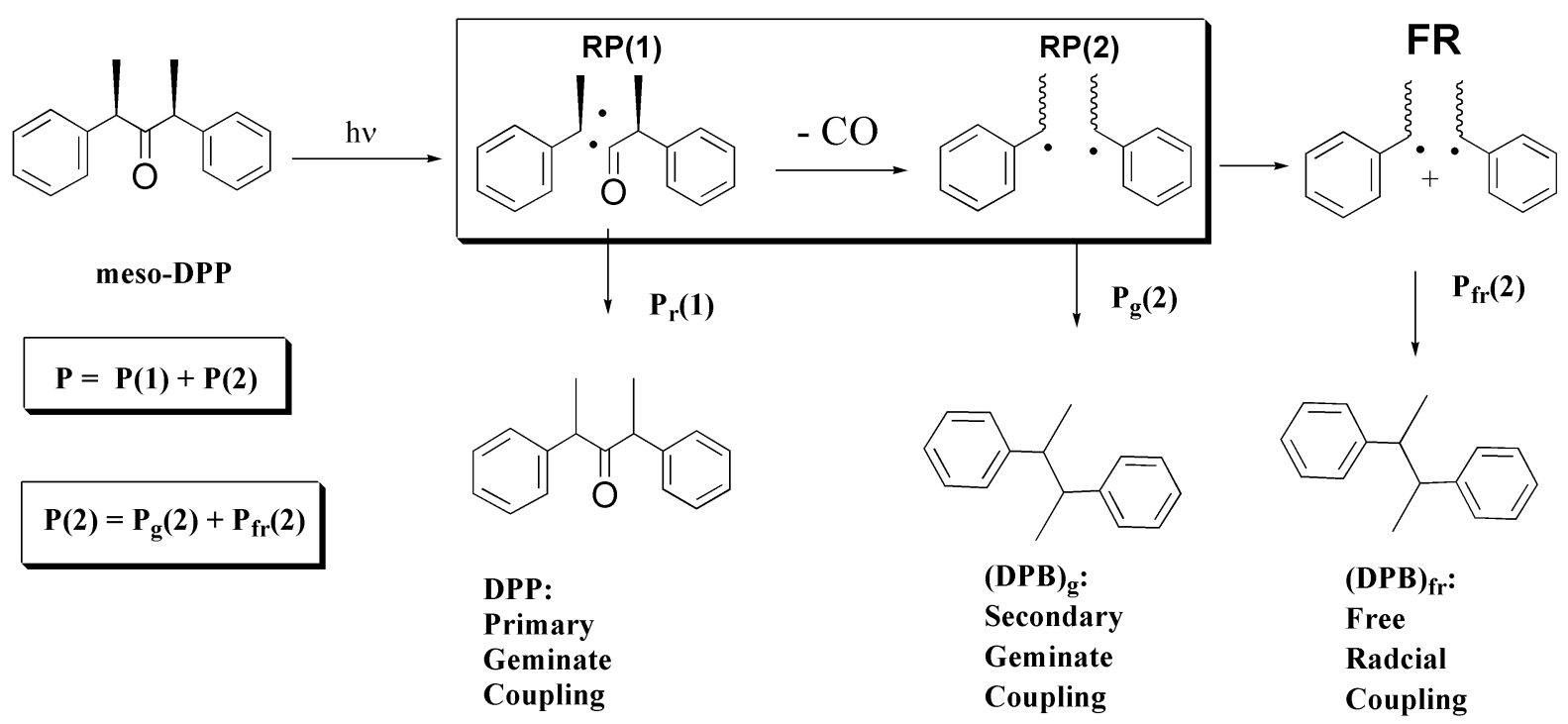

Scheme 2 Reaction mechanism and radical pair reaction pathways in the photochemistry of DPP. See text for discussion.

Can we employ supramolecular photochemistry to control the selectivity of product formation by steering the radical pairs involved in Schemes 1 and 2 down preselected pathways to the product the experimenter desires through the use of noncovalent, intermolecular interactions? What are the selectivities of radical-radical combination reactions that we might explore to control? They are (1) the geminatoselectivity (selectivity of geminate pair reactions versus irreversible free radical formation); (2) regioselectivity (fraction of ortho regioisomer to para regioisomer); (3) the chemioselectivity (selectivity of combination reactions of RP-1 versus the decarbonylation to form RP2). Since DPP possesses stereogenic centers, for this molecule we can also study (4) the stereoselectivity (selectivity of the stereochemistry of the recombination reactions for RP-1 and RP-2).

In addition to the control of radical pair reactivity by supramolecular effects, we shall introduce a novel selectivity control concept involving superdupermolecular non-covalent magnetic interactions to examine (5) the magnetoselectivity (influence of magnetic isotopes or applied magnetic fields on product formation) on the supramolecular photochemistry of DBK and DPP.

\section{Probabilities $(P)$ of product formation as mechanistic parameters}

The measurement of cage effects (\% geminate combinations) based on quantum yields are very difficult to measure for solids due to strong scattering and experimental uncertainties that are unavoidably introduced in any quantitative analysis. A simple, qualitative alternative that is related to the cage effect is, however, available. Based on simple product ratios, the probabilities, $P$, of reactions of the primary and secondary geminate pairs and free radicals at the various stages of reaction can be readily measured. The values of $P$ provide mechanistic insights that will be useful in design of supramolecular systems for which the selectivity of products can be controlled $(P$ is related to, but is not identical to, the conventional ${ }^{6}$ 'cage effect'). There is a certain probability of reaction, $P_{\mathrm{k}}(\mathrm{i})$, for each stage of the combination reactions of the primary (RP-1), secondary (RP-2) and free radical (FR) pairs shown in Schemes 1 and 2. The sum of the probabilities of all reactions starting from an initial geminate radical pair is 1.0. We define (Schemes
1 and 2) $P_{\mathrm{g}}(1)$ as the probability of all primary geminate pair recombination, and combination reactions, $P_{\mathrm{g}}(2)$ as the probability of all secondary geminate pair combination reactions, and $P_{\mathrm{fr}}$ as the probability of all free radical, random pair combination reactions (disproportionation is not a significant reaction in any of the systems investigated).

$$
\begin{gathered}
P=P(1)+P(2) \\
P(2)=P_{\mathrm{g}}(2)+P_{\mathrm{fr}}(2)
\end{gathered}
$$

Thus, the total probability of combination reactions, $P_{\mathrm{k}}(\mathrm{i})$ for $\mathrm{DBK}$ is given by

$$
P_{\mathrm{k}}(\mathrm{i})=P_{\mathrm{g}}(1)+P_{\mathrm{g}}(2)+P_{\mathrm{fr}}(1)+P_{\mathrm{fr}}(2)
$$

Experimentally, for both the DBK and DPP systems, $P_{\mathrm{fr}}(1)=$ 0 and is ignored in the analysis. In the case of DBK (Scheme 1) it is convenient to further decompose $P_{\mathrm{g}}(1)$ into the probability for recombination to form regioisomeric ortho and para isomeric ketones with probabilities, $P_{\mathrm{o}}(1)$ and $P_{\mathrm{p}}(1)$, respectively. Thus the total probability of all combination reactions after the formation of the initial primary geminate radical pair from DBK is given by eqn. (4).

$$
P_{\mathrm{k}}(\mathrm{i})=\left[P_{\mathrm{o}}(1)+P_{\mathrm{p}}(1)\right]+P_{\mathrm{g}}(2)+P_{\mathrm{fr}}(2)
$$

For DPP, the most significant primary combination is the reconnection of the bond that is broken by photolysis (Scheme 2) which involves the formation of a stereogenic center (regioisomers are formed only in low yields). We term this probability $P_{\mathrm{r}}$. Thus, the total probability of combination reactions, $\mathrm{P}_{\mathrm{k}}(\mathrm{i})$ for $\mathrm{DBK}$ is given by eqn. (5).

$$
P_{\mathrm{k}}(\mathrm{i})=P_{\mathrm{r}}(1)+P_{\mathrm{g}}(2)+P_{\mathrm{fr}}(2)
$$

From the measurement of $P_{\mathrm{k}}(\mathrm{i})$ and the interpretation of the products in terms of the $P$ parameters based on Schemes 1 and 2 , mechanistic information on the time evolution of the supramolecular radical pair is available. For example, for the DBK system, the geminatoselectivity (selectivity towards geminate combination) is given by the sum of $P_{\mathrm{g}}(\mathrm{i})$, the regioselectivity is given by the ratio $P_{\mathrm{o}}(1) / P_{\mathrm{p}}(1)$ and the chemioselectivity (selectivity towards primary combinations of $\mathrm{RP}-1$ relative to secondary combination of RP-2) is given by $P_{\mathrm{g}}(1) / P_{\mathrm{g}}(2)$.

We now apply Schemes 1 and 2 to examine the photochemistry of DBK@FAU and DPP@FAU, where FAU is a host 
zeolite of the faujasite family. ${ }^{18}$ At this point it is possible to propose a convenient working definition of supramolecular control and superdupermolecular control of selectivity: the experimental manipulation of the probabilities $P_{k}(i)$ to direct the system down a pathway that is selected through control of the supramolecular or superdupermolecular features of the system.

\section{Supramolecular photochemistry of DBK and DPP}

From the supramolecular standpoint the transformation from the gas phase to homogeneous solution to micelles to zeolites to crystals represents a continuous change in the ability of a host to serve as a constraining space for guest molecules, with the gas phase being a completely 'open' space to a compact crystal being a very 'hard' space. The supramolecular photochemistry of DBK and DPP has been thoroughly investigated in a range of host systems including micelles ${ }^{16,19}$ and zeolites. ${ }^{20}$ On one extreme of supercages, micelles provide a 'soft matter' host environment, ${ }^{16}$ whereas zeolites provide a 'hard matter' host environment. ${ }^{20}$ In this account we shall consider the supramolecular and superdupermolecular control of the chemistry of DBK and DPP as guests in the host supercages of the FAU family of zeolites. We now examine the structure of the DBK@FAU and DPP@FAU complexes whose photochemistry is the subject of this review.

\section{The host structure of FAU zeolites}

The FAU zeolite is a classical porous crystalline aluminosilicate $^{18}$ that occurs in nature. Two important synthetic forms of FAU are referred to as zeolite $\mathrm{X}$ and zeolite $\mathrm{Y}$. The unit cell composition of MX is $\mathrm{M}_{86}\left(\mathrm{AlO}_{2}\right)_{86}(\mathrm{SiO})_{106}$ and the unit cell composition of $\mathrm{MY}$ is $\mathrm{M}_{56}\left(\mathrm{AlO}_{2}\right)_{56}(\mathrm{SiO})_{136}$. So, although both MX and MY zeolites possess identical size supercages, they differ in their ratio of $\mathrm{Al}$ and $\mathrm{Si}$ atoms and, therefore, in the number of compensating metal cations. The $\mathrm{Al}$ and $\mathrm{Si}$ atoms are tetrahedrally bonded through oxygen atoms as shown in Scheme 3 to generate a framework of the zeolite's external and internal surface. This framework is very 'hard', since the atoms are linked in a crystalline array and are connected by very strong $\mathrm{AlO}$ and $\mathrm{SiO}$ bonds. From the standpoint of guest@host

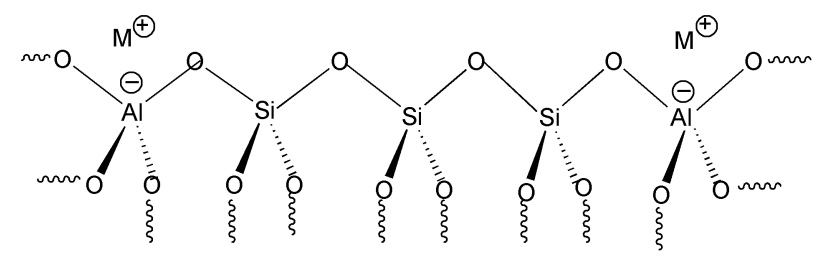

Scheme 3 Schematic representation of the composition of the framework of a FAU zeolite crystal.

chemistry, the size and shape of the void space generated by the framework is critical, since the pores on the external surface will serve to allow the sieving of molecules, based on their size and shape, and the void space of the supercages of the internal surface will provide the active sites for selectively 'controlling' the reactions of geminate radical pairs produced by photolysis of DBK@FAU and DPP@FAU complexes.

Scheme 4 shows a schematic of the framework's atoms of a FAU zeolite crystal and emphasizes the void space of the porous structure with framework composition $=\mathrm{M}_{x}\left(\mathrm{AlO}_{2}\right)_{x}\left(\mathrm{SiO}_{2}\right)_{y}$. Each line represents a bridging oxygen atom and each vertex represents an $\mathrm{Al}$ or $\mathrm{Si}$ atom of the composition. On the left of the Scheme is a representation of the external surface showing the roughly spherical pores ( $c a .8 \AA$ diameter) or holes that allow sieving into the internal surface and on the right is a representation of a single supercage which possesses a roughly spherical (ca. $13 \AA$ diameter) host void space.

\section{Supramolecular structure of DBK@FAU and DPP@FAU complexes}

We may change, at a fixed composition of guest and zeolite, the supramolecular structure of a guest@FAU complex by varying (1) the molecular structure of the guest molecules (examples, DBK and DPP), (2) the exchangeable cation density (number of cations per supercage, $\mathrm{X}$ versus $\mathrm{Y}$ ), and (3) the type of cation ( $\mathrm{Li}, \mathrm{Na}, \mathrm{K}$, etc). The value of the guest loading (\% wt guest $/ \%$ wt host) influences the supramolecular composition. The \% loading of the guest must influence the supramolecular structure of a guest@FAU complex and, therefore, is expected to influence the product distribution from photolysis of the

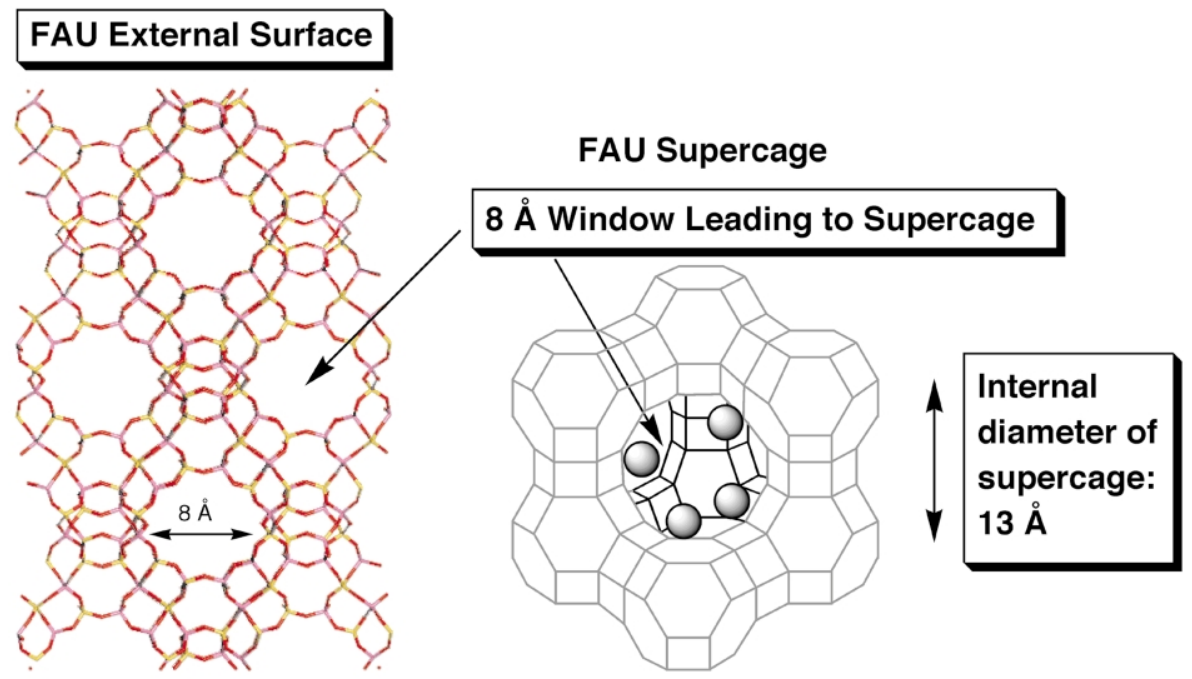

Scheme 4 Schematic of the void space of a FAU zeolite. Right: A view of the external surface. Note the pores leading to the internal surface are roughly circular and $\sim 8 \AA$ in diameter. Right. A view of a FAU supercage, which is roughly spherical and $\sim 13 \AA$ in diameter. For calibration, a benzene or naphthalene molecule can readily diffuse through the holes on the external surface, and the supercage can hold approximately 4-5 benzene molecules. Left: a view of the external surface showing the holes that provide windows to the internal supercages. Right: a view of a single supercage. The internal surface consists of a periodic array of supercages connected by the windows shown in the Scheme. 
complex. For example, at high loadings each supercage will possess a guest molecule. As a result, radical pairs will be blocked from diffusing throughout the adjacent supercages. At low loading diffusion throughout the porous framework will be much easier. Thus, results are best compared at fixed loading. Unless specified, the results reported below are all at comparable loading of guest molecules ( $\mathrm{ca}$. $2 \% \mathrm{wt} / \mathrm{wt}$, which corresponds to one guest molecule for every 3-4 available supercages on the average).

Even at a fixed loading for a given guest@FAU composition, there is still a supramolecular structural variation that is possible: addition of a co-guest to form a [guest/co-guest] @ host complex. Thus, there are a variety of supramolecular complexes that are possible for a given guest molecule and a given host zeolite framework through simple variations involving only 'non-covalent syntheses'. The richness and simplicity of these syntheses make investigations of products as a function of systematic variations of guest@zeolite supramolecular structure a fertile and exciting area for investigation.

Scheme 5 shows a schematic of a DPP molecule adsorbed in the supercage of $\mathrm{NaX}$ (left) and $\mathrm{NaY}$ (right), respectively. The

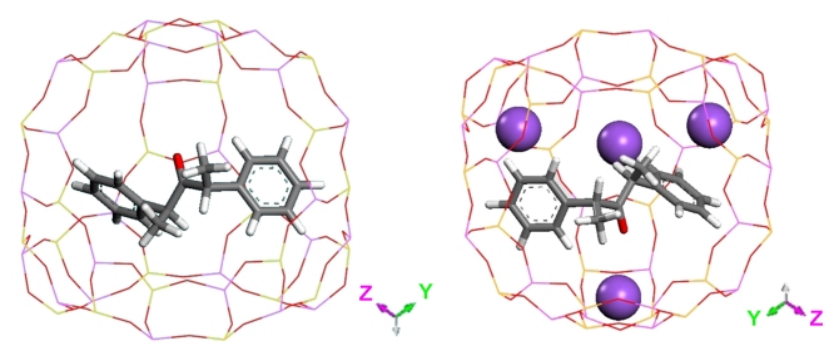

Scheme 5 Schematic representation of a DPP molecule in a FAU supercage. Right: a DPP molecule in a MX supercage showing several cations (balls) that occupy space within the supercage (some of the cations cannot find a nearby charge compensating negative $\mathrm{Al}$ atom to pair with. Left: a DPP molecule in a MY supercage showing no cations within the supercage (all the cations are securely bonded in the framework). The key point of the schemes is that there is more space in a MY supercage than there is in a MX supercage.

spheres indicate the mobile cations in the NaX supercage that are missing in the NaY supercage. In the MY zeolite the cations $^{21}$ are essentially all coordinated with oxygen atoms in the framework and are electrostatically 'pacified' to a certain extent (as a metal cation complexed to a crown ether) and are also not as sterically demanding as the cations in MX. The take home message from the Scheme is that there is considerably more space available to DBK and to the geminate pair produced by photolysis in a $\mathrm{NaY}$ supercage than there is in a $\mathrm{NaX}$ supercage.

\section{Structure-reactivity relationships in the supramolecular photochemistry of DBK@FAU and DPP@FAU}

Let us start by first inspecting the supramolecular photochemistry of DBK@FAU and then by considering the supramolecular structural variations that can be made and how these variations can control the resulting photochemistry, i.e., the probabilities for various reaction pathways (eqns. 1-5 and Schemes 1 and 2). In this analysis the guest molecular structure is considered fixed (for simplicity we ignore conformational considerations imposed by the complexation with the host, which can, however, be important). For the host structure, we are considering a single crystal of the zeolite framework (FAU), for which all of the empty supercages and topological connections between the supercages are periodically identical and fixed. We may systematically vary the composition $\left[\mathrm{M}_{x}\left(\mathrm{AlO}_{2}\right)_{x}\left(\mathrm{SiO}_{2}\right)_{y}\right]$ by varying the nature of the (monovalent) cation $\mathrm{M}($ e.g., $\mathrm{Li}, \mathrm{Na}, \mathrm{K}, \mathrm{Rb}, \mathrm{Cs})$ at fixed composition of $\mathrm{Al}$ or we may systematically vary the composition by fixing $M$ and varying the composition of $\mathrm{Al}$ ( $\mathrm{X}$ and $\mathrm{Y}$ zeolite).

The results 22 of the photochemistry of DBK@MX and of DBK@MY for $\mathrm{M}=\mathrm{Li}, \mathrm{Na}$ and $\mathrm{K}$ are summarized in Table 1 . $\mathrm{Rb}$ and Cs cations are not included for two reasons: (1) it is not possible to obtain $100 \%$ exchange of these ions in the composition so that one has at least two cations associated with the $\mathrm{Al}$ atoms of the framework when working with these cations, and (2) 'heavy atom' effects may begin to 'kick in' to complicate the intersystem crossing processes of the radical pairs. ${ }^{21}$

We now analyze the results in Table 1 (unless specified the loadings in the Tables correspond to $c a$. $2 \%$ weight of guest to weight of zeolite) by employing the $P_{\mathrm{k}}$ (i) parameters [eqn. (4)]. The value of $P_{\mathrm{g}}=\left[P_{\mathrm{g}}(1)+P_{\mathrm{g}}(2)\right]$, the sum of all primary geminate pair reactions $\left[P_{\mathrm{r}}(1)+P_{\mathrm{o}}(1)+P_{\mathrm{p}}(1)+P_{\mathrm{g}}(2)\right]$, reflects the 'cage effect' of primary and secondary geminate pair reactions. The yield of free radicals is given by $1-[P(1)+$ $P(2)]=P_{\mathrm{fr}}$. From Table 1 for photolysis of DBK@MX, there is a clear trend for the value of $P_{\mathrm{g}}$ to increase as the size of M is increased $(\mathrm{Li} \rightarrow \mathrm{Na} \rightarrow \mathrm{K})$. For example, as the size of the cation increases, geminatoselectivity (primary and secondary combinations) increases from $25 \%(\mathrm{Li})$ to $55 \%(\mathrm{Na})$ to $90 \%(\mathrm{~K})$. The cage effect for geminate combination of the secondary pair increases from 5\% to $30 \%$. Interestingly, although the $P_{\mathrm{g}}$ increases monotonically with the increasing size of $\mathrm{M}$ for MX, the cage effect is relatively constant and of the order of $c a .20 \%$, irrespective of M for MY.

Furthermore, the two regiochemistry parameters $P_{\mathrm{o}}(1)$ and $P_{\mathrm{p}}(1)$ can be measured as a function of supramolecular structure. As one proceeds from $\mathrm{M}=\mathrm{Li}$ to $\mathrm{M}=\mathrm{K}$, a clear trend in the regioselectivity parameter $P_{0}(1) / P_{\mathrm{p}}(1)$, the ratio of $o$ -

Table 1 Probability parameters for the photochemistry of DBK@FAU

\begin{tabular}{|c|c|c|c|c|c|c|c|}
\hline Parameter & $\begin{array}{l}\text { Primary } \\
\text { geminat }\end{array}$ & tivity & $\begin{array}{l}\text { Secondary } \\
\text { geminatoselectivity }\end{array}$ & $\begin{array}{l}\text { Free } \\
\text { radical } \\
\text { formation }\end{array}$ & $\begin{array}{l}\text { Total } \\
\text { geminatoselectivitye }\end{array}$ & $\begin{array}{l}\text { Regio } \\
\text { selectivity } f\end{array}$ & Chemioselectivitys \\
\hline \multicolumn{8}{|l|}{ System } \\
\hline & $P_{\mathrm{o}}(1)^{a}$ & $P_{\mathrm{p}}(1)^{b}$ & $P_{\mathrm{g}}(2)^{c}$ & $P_{\mathrm{f}}(2)^{d}$ & $P_{\mathrm{g}}(1)+P_{9}(2)$ & $P_{\mathrm{o}}(1) / P_{\mathrm{p}}(1)$ & $P(1) / P(2)$ \\
\hline DBK@LiX & $5 \%$ & $15 \%$ & $5 \%$ & $75 \%$ & $25 \%$ & 0.3 & 0.2 \\
\hline DBK@NaX & $20 \%$ & $25 \%$ & $10 \%$ & $45 \%$ & $55 \%$ & 0.8 & 0.8 \\
\hline DBK@KX & $40 \%$ & $20 \%$ & $30 \%$ & $10 \%$ & $90 \%$ & 2.0 & 1.5 \\
\hline DBK@LiY & $\sim 0 \%$ & $\sim 5 \%$ & $\sim 20 \%$ & $\sim 75 \%$ & $\sim 25 \%$ & - & 0.05 \\
\hline DBK@NaY & $\sim 0 \%$ & $\sim 5 \%$ & $\sim 20 \%$ & $\sim 75 \%$ & $\sim 25 \%$ & - & 0.05 \\
\hline DBK@KY & $\sim 0 \%$ & $\sim 5 \%$ & $\sim 20 \%$ & $\sim 75 \%$ & $\sim 25 \%$ & - & 0.05 \\
\hline
\end{tabular}

${ }^{a}$ Yield of $o$-MAP. ${ }^{b}$ Yield of $p$-MAP. ${ }^{c}$ Geminate combination to form DPE. ${ }^{d}$ Random combination to form DPE. $e$ Total primary and secondary geminate combination. $f$ Ratio of combinations to form $o$-MAP to combinations to form $p$-MAP. $g$ Ratio of combinations to form $[o$-MAP $+p$-MAP] to combinations to form DPE. 
MAP to $p$-MAP, monotonically increases from 0.2 to 1.5 . Finally, the ratio of primary geminate combinations to secondary geminate combinations, $P_{\mathrm{g}}(1) / P_{\mathrm{g}}(2)$, also increases monotonically from 0.3 to 2.0 as one proceeds from $\mathrm{M}=\mathrm{Li}$ to $\mathrm{M}=$ $\mathrm{K}$. Thus for the products from the photochemistry of DBK@MX complexes, the same trends are found for all of the parameters monitoring the relative tendency of combination to diffusion or decarbonylation: the larger the cation for the same number of cations or the larger the number of cations for the same cation, the greater the restriction on diffusional separation and intra-cage rotational motion of the geminate pairs.

At this point, let us consider the extent of control that has been achieved as the result of systematic variation of supramolecular structure for the DBK@MX system at 2\% wt/wt: (1) the geminatoselectivity has been increased from $25 \%$ for DBK@LiX to $90 \%$ for DBK@KX; (2) the regioselectivity increases for $o$-MAP formation from 0.3 to 2.0 upon going from DBK@LiX to DBK@KX; (3) the chemioselectivity for formation of regioisomers relative to decarbonylation products increases from 0.2 to 1.5 upon going from DBK@LiX to DBK@KX; (4) the cage effect for combination of the secondary geminate pairs increases from $5 \%$ to $30 \%$ upon going from DBK@LiX to DBK@KX.

It is expected that as the loading of DBK increases, the space available for diffusion of radicals between supercages will decrease and eventually at the highest loadings, the internal rotational motion of the radicals in a super cage will be affected. The value of the cage effect for DBK@ MX can be measured ${ }^{23}$ by using DBK that has one ring completely deuterated (DBK$\mathrm{d}_{5}$ ) and determining the ratio of the DPEs that are formed. The results of these studies are summarized in Table 2 which presents the cage effect as a function of loading. At low loadings $(0.4 \% \mathrm{wt} / \mathrm{wt})$ the cage effect is small $(5 \% \mathrm{wt} / \mathrm{wt})$, indicating efficient intersupercage diffusion. At higher loadings (5\% wt/ wt) the cage effect increases $(70 \%)$, indicating a significant decrease in the intersupercage diffusion. For DBK@MY the cage effect is not as sensitive to loading and only increases from
Table 2 Geminatoselectivity and DPE formation in the photolysis of DBK$\mathrm{d}_{5} @ \mathrm{FAU}$ as a function of loading of DBK

\begin{tabular}{|c|c|c|c|c|}
\hline $\begin{array}{l}\text { Loading } \\
\text { (wt/wt) }\end{array}$ & $\begin{array}{l}\text { DBK-d }_{5} @ \mathrm{NaX} \\
\text { Geminato- } \\
\text { selectivity }\end{array}$ & $\begin{array}{l}\text { DBK- } \\
\mathrm{d}_{5} @ \mathrm{NaX} \\
\text { Total DPE }\end{array}$ & $\begin{array}{l}\mathrm{DBK} \mathrm{d}_{5} @ \mathrm{NaY} \\
\text { Geminato- } \\
\text { selectivity }\end{array}$ & $\begin{array}{l}\text { DBK- } \\
\mathrm{d}_{5} @ \mathrm{NaY} \\
\text { Total DPE }\end{array}$ \\
\hline $0.4 \%$ & $5 \%$ & $85 \%$ & $10 \%$ & $98 \%$ \\
\hline $1.4 \%$ & $20 \%$ & $70 \%$ & $15 \%$ & $99 \%$ \\
\hline $3.0 \%$ & $35 \%$ & $35 \%$ & $20 \%$ & $80 \%$ \\
\hline $5.0 \%$ & $70 \%$ & $20 \%$ & $20 \%$ & $85 \%$ \\
\hline
\end{tabular}

$10 \%$ to $20 \%$ with a change in loading from $0.4 \%$ wt/wt to $5 \%$ wt/wt.

Increasing the loading of DBK is equivalent to increasing the probability of finding a guest molecule in the same supercage as a geminate radical pair produced by photolysis. Indeed, upon going from DBK@LiX to [DBK/benzene]@KX the cage effect for secondary radical combination increases from a value close to that for solution (4\%) to the highest value possible (100\%). Furthermore, the major product goes from DPE (80\%) to $o$ MAP (38\%) and the major regioisomer switches from $p$-MAP to $o$-MAP! It is also significant that the overall control of reaction selectivity is much less for DBK@NaY, presumably because of the greater free volume available in the $\mathrm{Y}$ supercages. ${ }^{23}$ After a mechanistic interpretation of the results, we shall show below how to take advantage of this "extra cargo space' available in NaY by filling it with a chiral shape to control the stereochemistry of combination reactions of the primary geminate radical pair.

\section{Mechanism of supramolecular control of geminate pair selectivity of the photochemistry of DBK@MX complexes}

A proposed mechanism that rationalizes all of the data in Table 1 and Table 2 is given in Scheme 6 and is based on the following ideas: (1) the products formed from the primary and secondary geminate pairs are determined by the relative rotational and

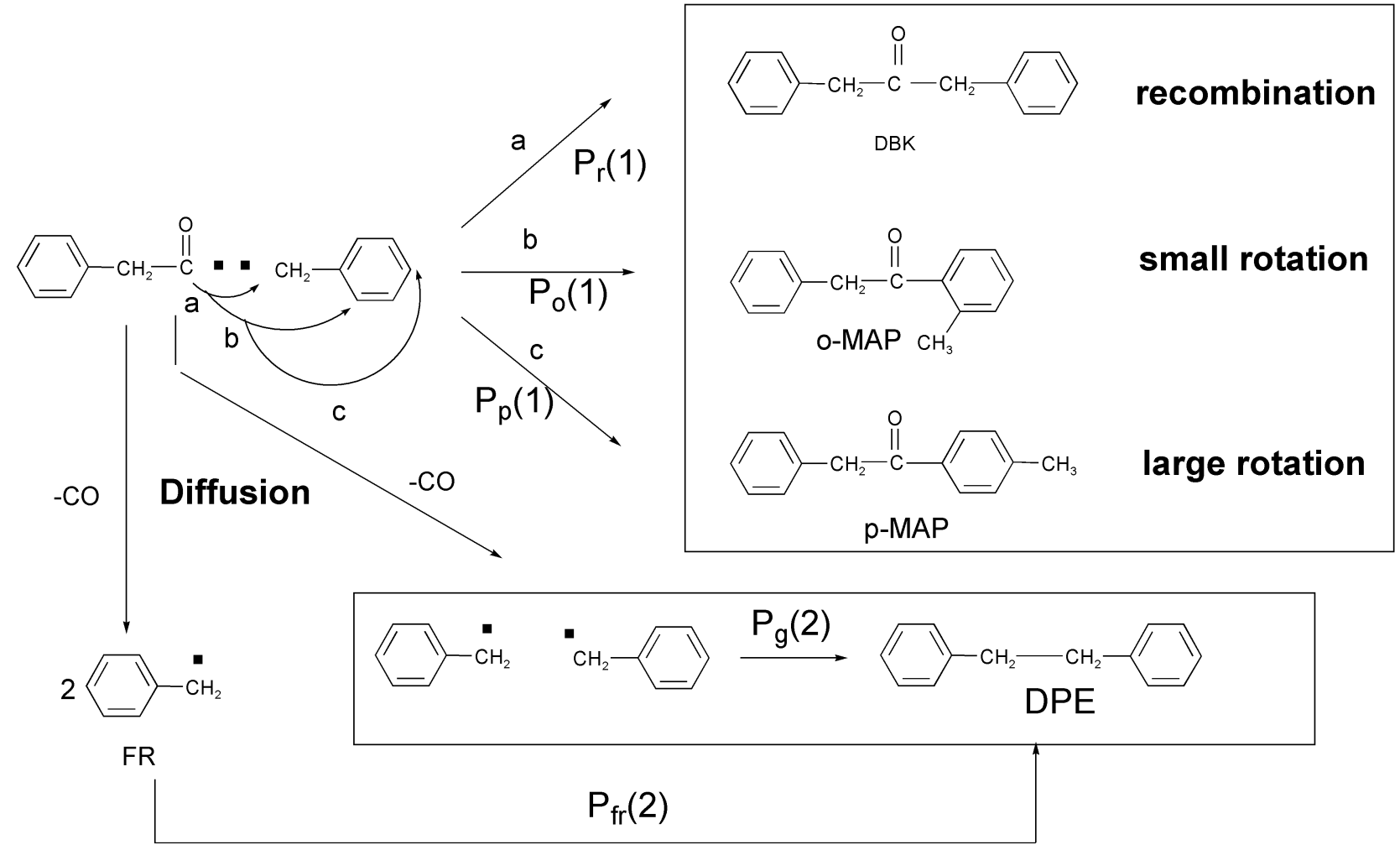

Scheme 6 Mechanism of supramolecular control of geminate pair reactions in supercages. See text for discussion. 
diffusional motions available to the pair and their competition with the rate of decarbonylation of the primary geminate pair; (2) the rate of decarbonylation is not significantly affected by the supramolecular structural variations; (3) the available free volume available to the geminate radical pairs determines the rate of relative rotation in the supercage; (4) the level of occupancy of the neighboring supercages determines the probability of diffusion of the pair out of the supercage to form free radicals. Although electrostatic effects are a priori expected to be involved and are known to be important in other cases, ${ }^{21}$ they do not appear to be important in the DBK@FAU systems investigated.

The regioselectivity of the recombination reactions of the primary pair to produce $o$-MAP and $p$-MAP (Scheme 6) provide information on the local rotational freedom experienced by the primary geminate pair in the supercage. The parameter $P_{\mathrm{o}}(1) /$ $P_{\mathrm{p}}(1)$, the ratio of the regioisomers $o-\mathrm{MAP} / p$-MAP, provides a measure of the local rotational motion: the greater the value of $P_{\mathrm{o}}(1) / P_{\mathrm{p}}(1)$, the greater the steric constraints on the relative rotational motion of the geminate pair (Scheme 6). From Table 1 it is seen that on going from $\mathrm{LiX}$ to $\mathrm{NaX}$ to $\mathrm{KX}$ the value of $P_{\mathrm{o}}(1) / P_{\mathrm{p}}(1)$ increases substantially from 0.3 to 2.0 , indicating that the relative rotational motion of the primary pair decreases as the size of $\mathrm{M}$ increases.

The chemioselectivity of product formation may be defined in terms of the ratio of primary pair coupling to the decarbonylation of the primary pair. The ratio of regioisomers to the decarbonylation product DPE $\left(P_{\mathrm{g}}(1) /\left[P_{\mathrm{g}}(2)+P_{\mathrm{fr}}(2)\right]\right)$ provides a measure of chemioselectivity, since it is a measure of the competition of coupling of the primary pair to the rate of decarbonylation. On going from $\mathrm{LiX}$ to $\mathrm{NaX}$ to $\mathrm{KX}$ the value of $P_{\mathrm{g}}(1) /\left[P_{\mathrm{g}}(2)+P_{\mathrm{fr}}(2)\right]$ increases from $20 \%$ to $60 \%$ indicating that the relative rate of pair coupling to decarbonylation increases as the size of $\mathrm{M}$ increases (this assumes that the rate of the unimolecular decarbonylation is less sensitive to the cation than the bimolecular coupling of the pair).

These results lead to the expectation that, since the free volume in MY zeolites is higher (fewer cations) relative to MX zeolites, there should be a higher degree of rotation and diffusion relative to decarbonylation. Indeed, the data in Table 1 for DBK@MY show that the geminatoselectivity and chemioselectivity, in contrast to the situation for DBK@MX, are relatively constant and independent of $\mathrm{M}$ ! Indeed, the major product (ca. 95\%) in all cases is DPE. The regioisomers $p$-MAP and $o$-MAP are formed in only trace amounts. These results are all completely consistent with the mechanism of Scheme 6: the supercage of the MY zeolites is 'open' to rotation and diffusion of the geminate pairs.

An interesting feature of the photolysis of the DBK@MY is the cage effect of $20 \%$ which is independent of M! A speculative explanation of this result is that the cage effect is determined not by free volume for DBK@MY complexes, but by statistical factors that determine the probability of escape of geminate secondary benzyl radicals from the supercage in which they are born. ${ }^{23}$
Another interesting feature of the results with DBK@NaY versus DBK@NaX is the effect of loading. For DBK@NaX the cage effect increases monotonically with increased loading from $\sim 5 \%$ at loadings less than $1 \%$ to very high cage effects (ca. 70\%) at very high loadings. On the other hand, for DBK@NaY, the cage effect is essentially fixed at $c a$. 10-20\% at all loadings. These results have been attributed to a faster diffusional motion in $\mathrm{NaY}$ that allows rapid exchange between supercages so that the cage effect is due only to statistical factors which are roughly independent of loading. ${ }^{23}$ Consistent with the more rapid combination rate as the space available for diffusional separation decreases is the observation that the total yield of DPE (geminate and free radical) decreases substantially with loading from $85 \%$ to $20 \%$ upon going from $0.4 \%$ loading to $5 \%$ loading.

\section{Superdupermolecular photochemistry: magnetic effects on the photochemistry of DBK@FAU}

The influence ${ }^{24}$ of magnetic isotopes and magnetic fields on the photochemistry of DBK@FAU is given in Table 3 for the DBK${ }^{12} \mathrm{CO} @ \mathrm{NaX}$ and DBK-13CO@NaX systems. The magnetic isotope effect on product selectivity was found to be most significant for $\mathrm{NaX}$ as a host compared to the other DBK@ MX or DBK@MY systems investigated, and constitutes an example of a dramatic superdupermolecular effect on the chemistry of geminate radical pairs. These results demonstrate the high sensitivity of magnetic effects to the supramolecular structure of the DBK@FAU systems.

The following trends are noted in Table 3: (1) the total primary geminatoselectivity $\left[P_{\mathrm{o}}(1)+P_{\mathrm{p}}(1)\right]$ increases sharply in going from DBK-12 C@NaX (44\%) to DBK-13 C@NaX (73\%); (2) the regioselectivity $\left[P_{\mathrm{o}}(1) / P_{\mathrm{p}}(1)\right]$ for formation of o-MAP relative to $p$-MAP increases significantly in going from DBK${ }^{12} \mathrm{C} @ \mathrm{NaX}(0.6)$ to $\mathrm{DBK}-{ }^{13} \mathrm{C} @ \mathrm{NaX}(1.0) ;$ (c) the chemioselectivity $[P(1) / P(2)]$ increases sharply in going from DBK${ }^{12} \mathrm{C} @ \mathrm{NaX}(0.8)$ to DBK-13 $\mathrm{C} @ \mathrm{NaX}$ (3.0). All of these effects are interpreted in terms of the decrease in the lifetime due to faster intersystem crossing of the primary pair containing ${ }^{13} \mathrm{C}$, i.e., because of the shorter lifetime, there is less time for decarbonation so the geminatoselectivity and chemioselectivity increases in favor of primary coupling products. Also, since there is less time for rotation of the primary radicals (see discussion in the next section), the regioselectivity increases in favor of the 'least motion' product, $o$-MAP.

There are smaller effects, close to the estimated experimental uncertainty, when an applied external magnetic field of $2000 \mathrm{G}$ is applied during the photolysis. In general, the trends observed with the applied field are opposite those for the magnetic isotope effects, i.e., the parameter $P_{\mathrm{o}}(1) / P_{\mathrm{p}}(1)$ decreases from 1.0 for the ${ }^{13} \mathrm{C}$ containing primary pair at zero field to 0.6 at 2000 G. Similar decreases in $P_{\text {tot }}(1) / P_{\text {tot }}(2)$ are observed when a magnetic field is applied. These effects are interpreted in terms of an increase in the lifetime of the primary pair due to slower intersystem crossing of the primary pair in the presence of a

Table 3 Magnetic effects on the photochemistry of DBK@NaX

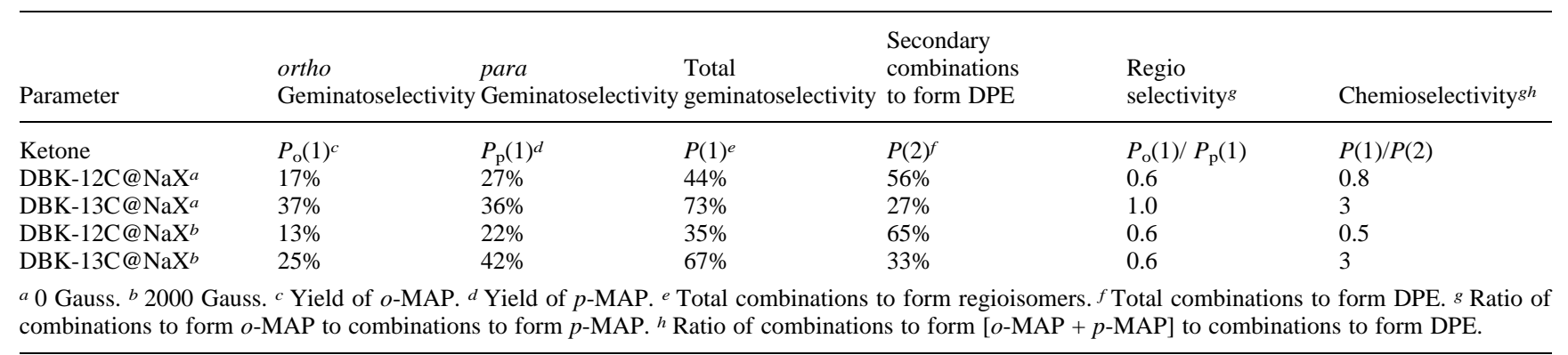


magnetic field, i.e., because of the longer lifetime, there is more time for rotation and decarbonylation so there is less $o$-MAP relative to $p$-MAP and more DPE relative to MAP (see discussion in the next section).

\section{The mechanism of superdupermolecular effects on the selectivity of reactions of geminate radical pairs}

The origin of the magnetic isotope effect and of the magnetic field effect on intersystem crossing (ISC) of geminate radical pairs in a supercage has been well established. ${ }^{19}$ Scheme 7 provides a brief description of the mechanistic basis for the geminatoselectivity, the regioselectivity and the chemioselectivity of product formation summarized in Table 3 . The ISC step converting a triplet geminate pair to a singlet geminate pair is responsible for the occurrence of spin effects in photoreactions in supramolecular systems. The key concept in spin chemistry is that the ISC step from the triplet pair to the singlet pair is a magnetic reactivity switch! This means that a triplet geminate radical pair cannot undergo a combination reaction until ISC occurs and a singlet is formed. This is due to the fact that spin selection rules forbid the triplet pair from directly forming singlet molecular products through radical-radical combination (or disproportionation) until ISC to a singlet state occurs.

The occurrence of a ${ }^{13} \mathrm{C}$ isotope at the carbonyl position of the primary pair increases ${ }^{19}$ ISC. Therefore, primary pair recombination to form regioisomers is enhanced because the ${ }^{13} \mathrm{C}$ isotope, being a magnetic nucleus, accelerates ISC in the geminate triplet pair through hyperfine coupling, whereas the ${ }^{12} \mathrm{C}$ isotope, being a non-magnetic isotope, cannot influence the rate of ISC. Faster formation of the singlet allows primary pair reactions to compete more favorably with both decarbonylation and diffusional and rotational motions of the pair. Thus, less DPE (diffusional separation product) and more regioisomers are produced when $\mathrm{DBK}-{ }^{13} \mathrm{CO} @ \mathrm{NaX}$ is photolyzed relative to DBK-12CO@NaX. Impressively, for the regioisomers, the relative amount of $o$-MAP (least rotational motion product) is significantly higher for DBK-13 $\mathrm{CO} @ \mathrm{NaX}$.

A qualitative model ${ }^{19}$ that integrates the concept of the supercage and spin chemistry was developed for triplet radical pairs in micelles and is shown in Fig. 2 (right). Just a brief description of the model is given here. For triplet geminate radical pairs in supercages whose size is of the order of $1 \mathrm{~nm}$ and for which the time scale of the ISC step is of the order of nanoseconds to microseconds, large spin effects due to applied

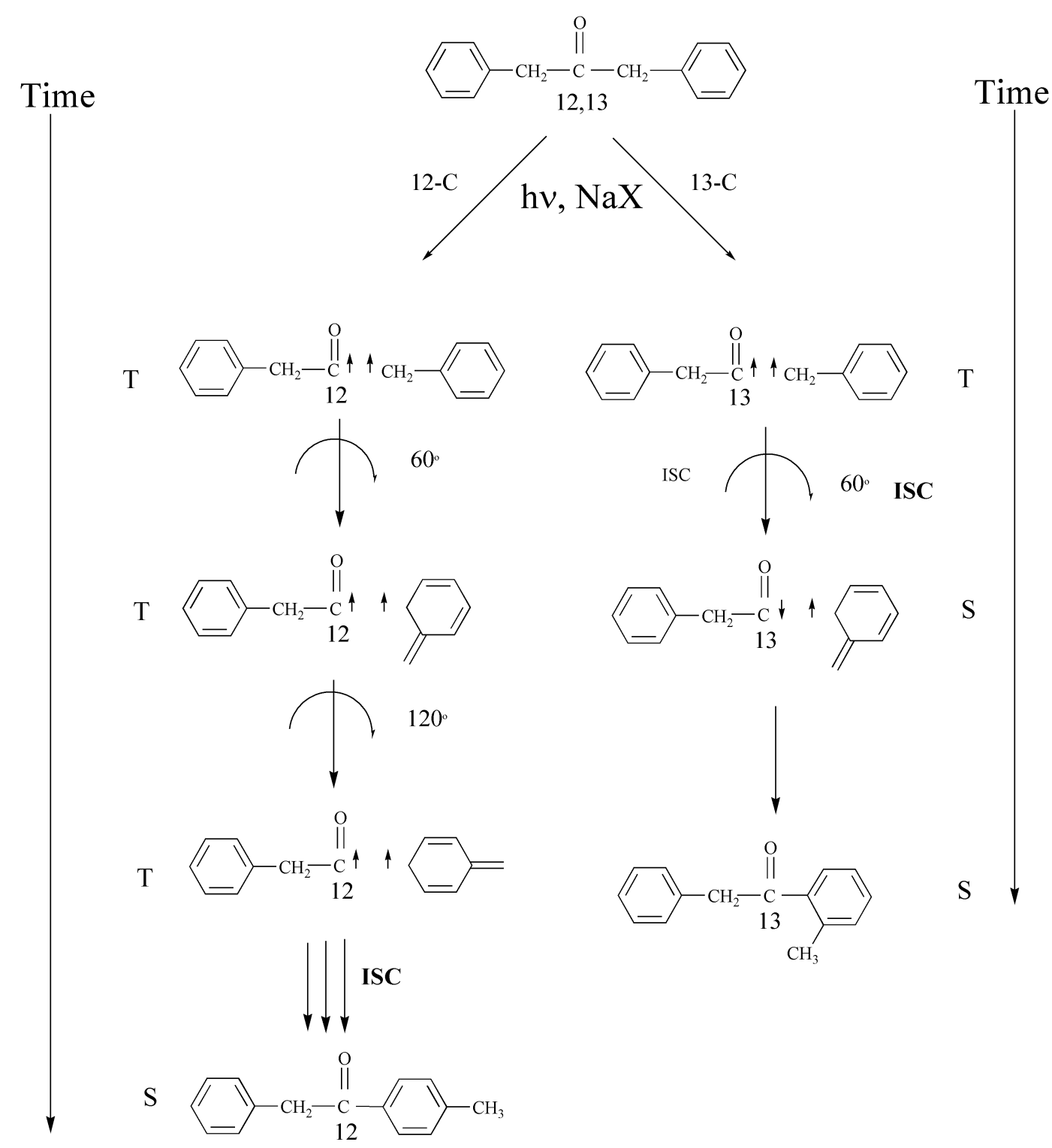

Scheme 7 Mechanism of ${ }^{13} \mathrm{C}$ isotope effect on geminate radical pair reactions. 
laboratory magnetic fields or to magnetic isotopes are expected theoretically ${ }^{19}$ and found experimentally ${ }^{16,19}$ on the value of the probability of recombination of an initially triplet geminate radical pair ('cage effects'). Since the size of the supercages of the FAU zeolites are of the order of $1 \mathrm{~nm}$, spin effects on the values of the cage effects for covalent bond formation between geminate triplet radical pairs are expected from theory.

A second important supramolecular aspect of the model is the intermolecular radical-radical interactions of electron exchange which play an important role in determining the magnitude of the observed spin effects. ${ }^{19}$ The exchange interaction between two radicals is a pure quantum mechanical supramolecular interaction which has no classical analogue! Thus, the combination of weak non-covalent supramolecular interactions between the radicals of a pair and weak exchange and magnetic interactions between the electron spins of the pair can control the reaction pathways of radical-radical reactions to selectively direct the formation of strong covalent bonds in supercages.

We apply the qualitative conclusions of the model for micelles to examine and understand the results of the magnetoselectivity of reactions in zeolites. As in the case of micelles, for the 'chemically inert' triplet geminate radical pair that is generated in the supercage of a zeolite, the time delay required for intersystem crossing to produce a 'chemically reactive' singlet geminate radical pair allows physical processes such as relative rotation and diffusion of the pair, or chemical processes such as decarbonylation and conversion of the pair to secondary radical pairs, to compete with recombination of the primary geminate pair (Scheme 7). An exciting conclusion from this model is the prediction that by using superdupermolecular (magnetic) effects to directly control the time of intersystem crossing and the lifetime of the pair, it should not only be possible to indirectly control the geminatoselectivity, regioselectivity and chemioselectivity of triplet geminate pair combinations, but it should also be possible to influence the stereoselectivity (enantioselectivity and diastereoselectivity) of primary geminate pair combinations.

The application of an external magnetic field reduces the probability of cage recombination of geminate pairs in micellar supercages, because ${ }^{19}$ the strong coupling of an external magnetic field with the electron spins inhibits the electron spins from undergoing ISC. This slows down the rate of singlet formation and allows the radicals to escape more efficiently out of the supercage, reducing the probability of the geminate pair combinations and produces exactly the opposite effects on selectivities as the ${ }^{13} \mathrm{C}$ isotope effect!

\section{Molecular photochemistry of DPP}

The photolysis of DPP in solution ${ }^{17}$ produces 2,3-diphenylbutane (DPB) in nearly quantitative yield (Scheme 2 ). Starting from pure meso-DPP, there is essentially no $(<0.5 \%)$ diastereoisomerization to form $d, l$-DPP and there is no diastereoselectivity in the formation of DPB. These results are consistent with the essentially quantitative diffusional separation of the geminate primary radical pair and quantitative decarbonylation of the random primary radical pair to produce random $\alpha$-methylbenzyl radicals, which in turn undergo essentially quantitative radical-radical combination. The cage effect for the photolysis of DPP was measured ${ }^{17}$ by determining the products from the photolysis of DPP- $\mathrm{d}_{6}$. A 1:2:1 mixture of DPB- $\mathrm{d}_{0}:$ DPB- $\mathrm{d}_{6}:$ DPB- $\mathrm{d}_{12}$ was observed indicating a cage effect of $\sim 0 \%$. Thus, the molecular photochemistry of DPP consists of the expected lack of significant geminate pair combination reactions from either the geminate primary or the geminate secondary pair.

\section{Supramolecular photochemistry of DPP@FAU}

In contrast to the results in solution, photolysis of DPP$\mathrm{d}_{6} @ \mathrm{NaX}$ or DPP- $\mathrm{d}_{6} @ \mathrm{NaY}$ results only in formation of DPB- $\mathrm{d}_{6}$, and $n o D P B-d_{0}$ or $D P B-d_{12}$ are formed indicating a geminatoselectivity of $100 \%$ for combination of secondary geminate radicals (Table 4 ). This result demonstrates that the supercages

Table 4 Stereoselectivity in the photolysis of [meso-DPP/ephedrine] @ NaY

\begin{tabular}{llll}
\hline NaY & $d, l$-DPP* & DPB & ee \\
\hline (-)-Ephedrine & & & \\
12C (0G) & $39 \%$ & $61 \%$ & +3.6 \\
13C (0G) & $48 \%$ & $52 \%$ & +7.1 \\
12C (2000G) & $42 \%$ & $58 \%$ & +4.3 \\
(+)-Ephedrine & & & \\
12C (0G) & $24 \%$ & $76 \%$ & -5.1 \\
13C (0G) & $28 \%$ & $72 \%$ & -7.3 \\
12C (2000G) & $35 \%$ & $65 \%$ & -3.6 \\
No Inductor & & & \\
$12 \mathrm{C}(0 \mathrm{G})$ & $27 \%$ & $64 \%$ & - \\
$13 \mathrm{C}(0 \mathrm{G})$ & $54 \%$ & $44 \%$ & - \\
\hline
\end{tabular}

of $\mathrm{NaX}$ and $\mathrm{NaY}$ severely constrain the diffusional separation of the geminate secondary radicals produced by photolysis of DPP, but that a certain degree of separation of the geminate primary radical occurs and that decarbonylation to generate the geminate secondary pair is efficient during the period of separation of the primary pair. These results should be compared to the situation for the geminate primary radical pair produced from photolysis of $\mathrm{DBK} @ \mathrm{NaX}$, for which a considerably fraction $(\mathrm{ca} .50 \%)$ of the geminate primary pair undergoes combination before decarbonylation (Table 1). The geminate primary pair produced from DBK is expected to separate faster (because of the smaller size of the pair) than the geminate primary pair produced from DPP and to decarbonylate slower than the geminate radical pair from DPP. Thus, the relatively higher fraction of geminate primary pair combination in the case of DPP@FAU can be attributed to either the slower rate of decarbonylation ${ }^{15}$ of the $\mathrm{PhCH}_{2} \mathrm{CO}$ radical compared to the $\mathrm{PhCH}\left(\mathrm{CH}_{3}\right) \mathrm{CO}$ radical (in solution the latter radical decarbonylates at a rate $c a .8$ times faster than the former radical) or to a slower diffusional rate of coupling of the $\mathrm{PhCH}_{2} \mathrm{CO}$ radical relative to the $\mathrm{PhCH}_{2} \mathrm{CO}$ radical (or a combination of both).

Photolysis of pure meso-DPP@NaX or meso-DPP@NaY produces a significant (conversion dependent) amount of $d, l$ DPP (in solution, $<0.5 \% d, l$-DPP is formed) and a low yield of regioisomers. In addition, in the case of meso-DPP@NaX there is a diastereomeric selectivity for $d, l$-DPB over meso-DPB ( $c a$. $2: 1$, de $=30 \%)$.

\section{A new mechanistic parameter, $P_{\mathrm{r}}$, the probability of recombination of the primary geminate pair}

The products of the photochemistry of DPP@FAU are analogous to those of DBK@FAU (Scheme 2). However, there are several important structural and kinetic differences. Structurally, because DPP exists as three stereoisomers ( $d$-DPP, $l$ DPP and $m$-DPP), the reformation of the initial bond which is broken by $\alpha$-cleavage may now be monitored directly by measuring the extent of stereochemical interconversion (meso $\rightarrow d, l$ or $d, l \rightarrow m e s o$ ). The extent of stereointerconversion as a function of conversion allows the computation of a new parameter, $P_{\mathrm{r}}(1)$, the probability of recombination of the primary geminate radical pair. This parameter may be compared to the extent of regioisomer formation as a function of supramolecular structure to provide information on the relative local rotation of the primary pair. 
In general, stereoselectivity in the recombination reactions of radical pairs in solution is very low, because a prochiral, flexible carbon radical center is typically incapable of retaining configuration during the time scale of diffusional excursions between the creation of the pair and the combination reactions of free radicals. ${ }^{25} \mathrm{We}$ sought to design a guest/(chiral coguest)@zeolite system whose photolysis would lead to enantiomeric selectivity in the recombination of the geminate pair produced by photolysis. There were two important requirements for such a system: (1) the separation of the primary geminate pair must be allowed to occur so that a significant diastereomeric interaction can occur between the prochiral geminate pair and the chiral inductor co-guest, but there should be a high probability of geminate recombination; (2) the geminate recombination must be enantiomerically selective because of the interactions of the prochiral pair with the co-guest chiral inductor.

If the process of formation and recombination of the geminate radical pair is reversible, the probability of recombination $\left(P_{\mathrm{r}}\right)$ may be determined experimentally by starting with a stereopure guest and then measuring the extent of stereoisomerization as a function of conversion. ${ }^{26}$ If a plot of (the $\log$ of) the loss of stereochemistry versus (the log of) conversion is a straight line, the slope of the line directly provides the value of $P_{\mathrm{r}}$. Experimentally, ${ }^{27}$ this is found to be the case for the photolysis of meso-DPP@NaY. The value of $P_{\mathrm{r}}$ is 0.15 implying a significant degree of reversibility in the recombination reaction of the primary geminate radical pair produced by photolysis of meso-DPP@NaY.

\section{Superdupermolecular effects in the photolysis of DPP@NaY}

The products of the photochemistry of DPP@FAU, similar to the products of the photochemistry of DBK@FAU, encode information on supramolecular structure and dynamics of the pathway from the starting reactant through intermediates and finally to the product. Photolysis of meso-DPP@NaY produces $d, l$-DPP, regioisomers of DDP, meso-DPB and $d, l$-DPB as the most significant products (Table 5). The parameter $P_{\mathrm{r}}$ (which is determined by a measurement of the extent of meso $\rightarrow d, l$ conversion as a function of extent of photolysis ${ }^{26,27}$ ) provides a measure of the probability of return of the primary geminate radical pair. The role of magnetic isotopes, a spin or superduper molecular effect is expected for the probability of the combination of the radical pair, because the rate of intersystem crossing is expected to be faster for the ${ }^{13} \mathrm{C}$ containing pair than for the ${ }^{12} \mathrm{C}$ containing pair.

The value of $P_{\mathrm{r}}$ is $c a$. 0.04 for photolysis of DPP in homogeneous solution, ${ }^{7}$ i.e., just a few percent of the primary geminate pair produced by photolysis recombine, and almost all of the geminate pairs diffuse apart and become free radicals. On the other hand, ${ }^{28} P_{\mathrm{r}}$ is 0.15 for meso-DPP-12C@NaY, indicating that a significant fraction of geminate pairs undergoes recombination. The value of $P_{\mathrm{r}}$ jumps to 0.20 for meso-DPP${ }^{13} \mathrm{C} @ \mathrm{NaY}$. This remarkable $40 \%$ change in $P_{\mathrm{r}}$ as a function of ${ }^{12} \mathrm{C}$ and ${ }^{13} \mathrm{C}$ isotopes is a superdupermolecular spin effect on geminate pair recombination and is due to the more rapid intersystem crossing in the primary geminate pair that is induced by the strong ${ }^{13} \mathrm{C}$ hyperfine coupling of the carbonyl radical center. The significant isotope effect is manifested in the yields of $d, l$-DPP among the photolysis products (Table 5). For example, for comparable conversions, the yield of $d, l$-DPP from photolysis of meso-DPP-12C@NaY is 27\% and for photolysis of meso-DPP-13C@NaY the yield is 54\% of the product mixture. The yield of secondary coupling products, DPB decreases from $64 \%$ for meso-DPP-12C@NaY to $44 \%$ for meso-DPP13C@NaY.

\section{Supramolecular chiral auxiliaries. Superdupermolecular effects on enantiomeric selectivity}

A number of investigations have demonstrated that zeolites may serve as hosts for enantiomeric selectivity in photochemical

Table 5 Magnetoselectivity in the photolysis of [meso-DPP/ephedrine]@NaY<smiles>C[C@H](P)C(=O)[C@@H](N)[Pb]</smiles>

meso-DPP- $-{ }^{12} \mathrm{C}$ meso-DPP- $-{ }^{13} \mathrm{C}$

\section{Magnetoselectivity \\ of recombination probability, $\mathbf{P}_{\mathbf{r}}$ :}

$$
\begin{aligned}
& \mathrm{P}_{\mathrm{r}}{ }^{13} \mathrm{C}=0.20 \\
& \mathrm{P}_{\mathrm{r}}{ }^{12} \mathrm{C}=0.15
\end{aligned}
$$

Magnetoselectivity of product selectivities.

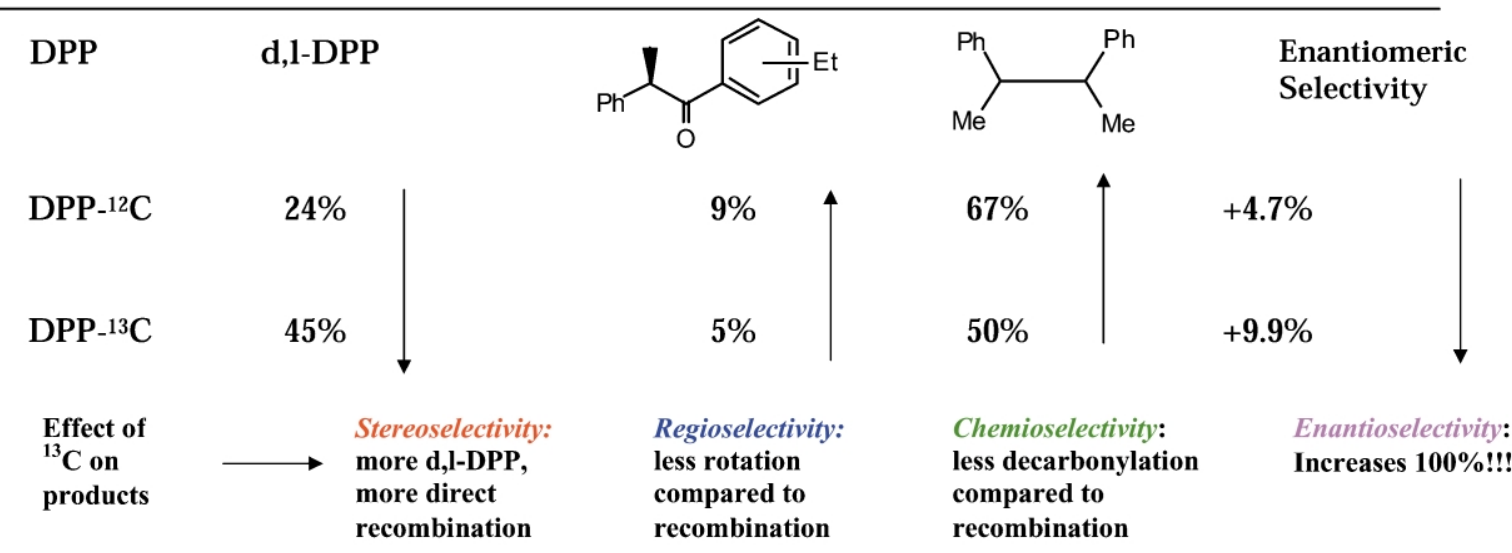


processes. ${ }^{29}$ Of particular interest in this report is the use of ee as a mechanistic probe of the supramolecular structure and dynamics. After a survey of a number of potential candidates, the [meso-DPP/ephedrine]@NaY system emerged (Scheme 8)

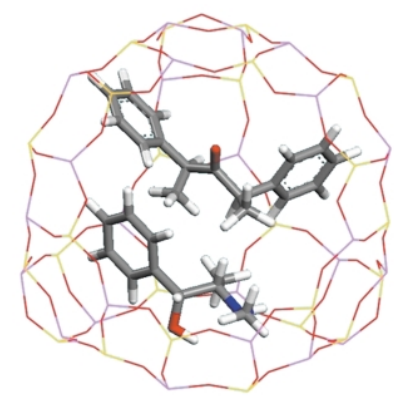

Scheme 8 Schematic representation of [DPP/ephedrine]@NaY. This representation should be compared to those in Scheme 5 for DPP@NaX and DPP@NaY.

as an attractive candidate for investigating the mechanism of enantioselectivity of geminate radical pairs. ${ }^{30}$

A particularly interesting feature of the photochemistry of DPP@FAU involves the possibility of chiral induction for systems of the type [DPP/ChiralGuest]@FAU. The concept here is that if DPP and a chiral guest molecule occupy the same supercage, the primary radical pair produced by photolysis, which is prochiral, may recombine with a certain degree of enantiomeric selectivity. The degree of selectivity will depend on the fraction of cages that contain both a chiral inductor and DPP and the supramolecular structure and dynamics of those supercages. In addition, if enantiomeric selectivity is observed, it is expected to be sensitive to a magnetic isotope carbon effect, since $P_{\mathrm{r}}$ is significantly sensitive to the presence of ${ }^{12} \mathrm{C}$ or ${ }^{13} \mathrm{C}$ at the carbonyl position. Indeed, these expectations are confirmed ${ }^{30}$ by experiment (Table 5): with (+)- or (-)-ephedrine as co-guest in the photolysis of [meso-DPP/ephedrine]$@ \mathrm{NaY}$ the $d, l$-DPP produced is enantiomerically enriched. Photolysis of [meso-DPP/(-)-ephedrine]@NaY yields an excess of (-)-d,l-DPP and photolysis of (meso-DPP/(+)-ephedrine)@NaY yields an excess of (+)- $d, l$-DPP

Perhaps the most remarkable finding 30 is that for photolysis of [meso-DPP-13 $\mathrm{C} /(-)$ ephedrine] $@ \mathrm{NaY}$, relative to [mesoDPP-12C /(-)-ephedrine]@ NaY, the enantiomeric excess increases by $c a$. $100 \%$ ! As described above for DBK, the magnetic isotope effect is attributed to an increase of ISC in the primary geminate radical pair. The shorter lifetime of the primary pair evidently is optimized for interactions of the prochiral pair with the chiral inductor. Application of a magnetic field of $2000 \mathrm{G}$ does not significantly influence the extent of ee for the meso-DPP-12 $\mathrm{C} /(-)$-ephedrine system.

The results of photolysis of [meso-DPP-12C/(-)-ephedrine]@NaY and [meso-DPP-DPP-13C/(-)-ephedrine]@ NaY presented in Table 5 emphasize the various selectivities that are possible with superduper effects. Note that in each case the ${ }^{13} \mathrm{C}$ enhances the formation of products that are favored by more rapid ISC, namely $\mathrm{Pr}$, recombination relative to regiocombination, $O$-regioselectivity relative to $p$-regioselectivity, and chemioselectivity for combination relative to decarbonylation. There was no a priori reason for expecting the selectivity of ee to increase or decrease when ${ }^{13} \mathrm{C}$ was substituted for ${ }^{12} \mathrm{C}$, only that there should be a change.

\section{Conclusion}

Molecular chemistry is concerned with the covalent bond and the molecular structure resulting from covalent connections. Effectively, molecular chemistry in solution is gas phase chemistry modified by 'structureless' properties of the solvent that determines viscosity or polarity. The 'solvent cage' about a molecule or pair of molecules or pair of radicals provides the vestiges of non-covalent interactions that are at the heart of supramolecular chemistry, which is defined as chemistry dealing with intermolecular interactions beyond the molecule and the covalent bond. To date the field of supramolecular chemistry has been concerned with intermolecular electrostatic interactions resulting in the formation of weak intermolecular bonds and supermolecules and supramolecular assemblies in their singlet ground state. Supramolecular photochemistry takes supramolecular chemistry beyond the singlet ground state and introduces the complexity and richness of control of chemistry by electronically excited states surfaces and of triplet state surfaces.

Triplet surfaces introduce the possibility of superdupermolecular chemistry for which magnetic properties exert significant control over chemical reactivity and selectivity. In particular, the magnetic properties of triplet radical pairs introduces the possibility of controlling radical pair reactivity and selectivity through magnetic effects due to magnetic nuclear spins and external magnetic fields. These magnetic effects are manifest commonly and strongly in supramolecular systems of the size of nanometers because of the coincidence of the time scales of hyperfine induced ISC and the diffusional dynamics of radical pairs in restricted spaces (Fig. 2, right). Running reactions in nanoscopic reactors hold promise for controlling reaction selectivity in the formation of covalent bonds through supramolecular effects. In this sense "inert" nanoscopic reactors are catalysts or reagents that control the selectivity of formation of possible products. In collaboration with photons as reagents to form triplet radical pairs, [guest/ photon]@host and [guest/co-guest/photon]@host systems hold great promise as an extension of the arsenal of synthetic organic chemistry in addition to providing a rich field for mechanistic investigation.

Fig. 1 summarizes the progress that has been made on the theory of the molecular cage effect (left) and indicates a modern interpretation (right) in terms of energy surfaces that include spin effects. The dissociative surface which produces the geminate radical pair is now identified as a triplet state, and the boundary that inhibits the separation of the geminate pair is the wall of the supercage which produces the supramolecular effects on the geminate pair reactivity. The requirement for ISC produces superdupermolecular effects by serving as a spin and magnetic field sensitive switch for combination reactions of the pair. The acceleration or deceleration of ISC changes the competition between the rate of combination reactions and the mechanical process of rotation and diffusion of the primary geminate pair that controls the enantioselectivity and regioselectivity of the combination reactions of the primary geminate pair. The acceleration or deceleration of ISC also changes the competition between the rate of decarbonylation and combination of the geminate radical pair, that controls the chemioselectivity of the reaction. Fig. 2 summarizes the progress that has been made in the theory of the supramolecular cage effect. On the left, is the mechanical model which revealed that in supramolecular systems, collisions occur in sets; on the right, is the modern interpretation which shows how a random walk of the random pair from an origin coupled with the operation of spin dynamics during the walk determine the probability of geminate combination when the pair returns to the origin, and lead to a natural origin of magnetic effects on the combination reactions of geminate radical pairs.

The combination of supramolecular and superdupermolecular effects demonstrates the power of controlling covalent bond formation in radical pairs through non-covalent intermolecular and magnetic interactions. The examples presented in this report demonstrate the ability of weak interactions, when 
judiciously applied, to control the selectivity of covalent bond formation.

\section{Acknowledgments}

The author thanks his colleagues and students for providing enormous stimulation and excitement in developing the ideas and performing the experiments presented in this review. Professor Anatolii L. Buchachenko has been a muse for the appreciation and understanding of the beauty of spin chemistry. Dr Valery F. Tarasov was an excellent tutor for the quantitative understanding of spin effects on geminate pair reactivity. Our work on the photochemistry of molecules adsorbed on zeolites was inspired by two industrial mentors, Dr Edith Flanigen and Dr Lloyd Abrams. The ground breaking research on controlling combination reactions of geminate radical pairs for the DBK@FAU and DPP@FAU systems discussed in this review was performed by Miguel Garcia-Garibay, Zhenyu Zhang, XueGong Lei and Naresh Ghatlia. The research on enantioselectivity of geminate radical pair recombinations was performed by Nikolaos A. Kaprinidis, Margaret Landis and George Lem. Professor V. Ramamurthy's research inspired the experiments involving enantiomeric selectivity. Special thanks go to Professor Thomas Poon and Dr L. Pranatharthiharan for their excellent assistance in producing the graphics used for the cover and some of the Schemes. This research has been generously supported by the National Science Foundation for over two decades. Most recently, this research was supported by grant NSF CHE 01-10655 and in part by the National Science Foundation and the Department of Energy under grant NSF CHE 98-10367 to the a Environmental Molecular Science Institute at Columbia.

\section{Notes and references}

1 J. Franck and E. Rabinowitch, Trans. Faraday Soc., 1934, 30, 120. 2 (a) J.-M. Lehn, Supramolecular Chemistry, 1995, VCH, New York; (b) J.-M. Lehn, Angew. Chem., Int. Ed. Engl., 1988, 27, 89; (c) J.-M. Lehn, Science, 1985, 227, 849; (d) J. W. Steed and J. L. Atwood, Supramolecular Chemistry, Wiley, NY, 2000.

3 D. J. Cram and J. M. Cram, Container Molecules and Their Guests, Royal Society of Chemistry, Cambridge, UK, 1994.

4 E. Rabinowitch and W. C. Wood, Trans. Faraday Soc., 1936, 32, 1381.

5 R. M. Noyes, J. Am. Chem. Soc., 1955, 77, 2042.

6 (a) T. Koenig and H. Fischer, Free Radicals, Wiley, New York, 1973; (b) J. P. Lorand, Inorganic Reaction Mechanisms, ed. J. O. Edwards, NY, Interscience, New York, NY, 1972.
7 E. N. Step, A. L. Buchachenko and N. J. Turro, J. Org. Chem., 1992, 57, 7018.

8 L. Pauling, The Nature of the Chemical Bond, Cornell University Press, Ithaca, NY, 1954.

9 N. J. Turro, Angew. Chem., Int. Ed. Engl., 1986, 25, 882.

10 L. Stryer, Biochemistry, W. H. Freeman., San Francisco, 1981.

11 J. Retey, Angew. Chem., Int. Ed. Engl., 1990, 29, 355; J. Stubbe and W. A. van der Donk, Chem. Rev., 1998, 98, 705.

12 (a) N. Herron, Chemtech., 1989, 542; (b) R. D. Parton, D. De Vos and P. A. Jacobs, Zeolite Microporous Solids: Synthesis, Structure and Reactivity, eds. E. G. Derouane, F. Lemos, C. Naccache and F. R. Ribeiro, Kluwer Academic Publishers, Dordrecht, The Netherlands, 1992, p. 555-578.

13 (a) P. B. Weisz, Ind. Eng. Chem. Fundam., 1986, 25, 53-58; (b) S. M. Csiscery, Zeolites, 1984, 4, 202.

14 DBK photochemistry: (a) P. S. Engel, J. Am. Chem. Soc., 1970, 92, 6074; (b) W. K. Robbins and R. H. Eastman, J. Am. Chem. Soc., 1970, 92, 6076; (c) DPP photochemistry: B. H. Baretz and N. J. Turro, J. Am. Chem. Soc., 1983, 104, 1309; (d) Diffusion controlled reactions of radicals: R. F. C. Claridge and H. Fischer, J. Phys. Chem., 1983, 87, 1960 .

15 (a) L. Lunazzi, K. U. Ingold and J. C. Scaiano, J. Phys. Chem., 1983, 87, 529; (b) N. J. Turro, I. R. Gould and B. Baretz, J. Phys. Chem., 1983, 87, 531.

16 N. J. Turro and G. C. Weed, J. Am. Chem. Soc., 1983, 105, 1861.

17 N. D. Ghatlia and N. J. Turro, J. Photochem. Photobiol. A: Chem., 1991, $\mathbf{5 7}, 7$.

18 (a) D. W. Breck, Structure, Chemistry and Use, Wiley, NY, 1974; (b) L. Abrams and D. R. Corbin, J. Inclusion Phenom. Mol. Recognit. Chem., 1995, 21, 1 .

19 (a) N. J. Turro and B. Kraeutler, Acc. Chem. Res., 1980, 13, 369; (b) N. Turro, A. L. Buchachenko and A. F. Tarasov, Acc. Chem. Res., 1995, 28 , 69; (c) A. L. Buchachenko and E. L. Frankevich, Chemical Generation and Reception of Radio- and Microwaves, VCH, New York, NY, 1994.

20 N. J. Turro, Acc. Chem. Res., 2000, 33, 637.

21 V. Ramamurthy and N. J. Turro, J. Inclusion Phenom. Mol. Recognit. Chem., 1995, 21, 238.

22 (a) N. J. Turro and Z. Zhang, Tetrahedron Lett., 1987, 28, 5637; (b) Z. Zhang, PhD Thesis, Columbia University, 1989.

23 (a) M. A. Garcia-Garibay, Z. Zhang and N. J. Turro, J. Am. Chem. Soc., 1991, 113, 6212; (b) M. A. Garcia-Garibay, X. G. Lei and N. J. Turro, J. Am. Chem. Soc., 1992, 114, 2749.

24 N. J. Turro and Z. Zhang, Tetrahedron Lett., 1989, 30, 3761.

25 (a) K. R. Kopecky and T. Gillan, Can. J. Chem., 1969, 47, 2371; (b) F. D. Greene, M. A. Berwick and J. C. Stowell, J. Am. Chem. Soc., 1970, 92, 867.

26 V. F. Tarasov, B. B. Klimenok and A. Buchachenko, Bull. Acad. Sci. USSR (Engl. Transl.), 1987, 36, 500.

27 N. A. Kapinidis, M. S. Landis and N. J. Turro, Tetrahedron Lett., 1997, 38, 2609.

28 G. Lem, Columbia University, unpublished results.

29 A. Joy and V. Ramamurthy, Chem.-Eur. J., 2000, 6, 1287.

30 G. Lem and N. J. Turro, Chem. Commun., 2000, 293. 